Series A

I. MATHEMATICA

594

\title{
PONTRJAGINRÄUME \\ MIT EINEM REPRODUZIERENDEN KERN
}

VON

PEKKA SORJONEN

H E L S N K I 1975

S U O M A L A I N E N T E D E A K A T E M I A

doi:10.5186/aasfm.1975.594 
Copyright (C) 1975 by

Academica Scientiarum Fennica

ISSN 0066-1953

ISBN 951-41-0210-X

Vorgelegt am 13. Mai 1974 


\section{Einleitung}

0.1. In dieser Note wird die von N. Aronszajn, S. Bergman und M. G. Kreı̌n entwickelte Theorie der Hilberträume mit einem reproduzierenden Kern (siehe [1], [2], [6] und weitere in [11] zitierte Arbeiten) in zwei Richtungen verallgemeinert: Anstatt komplexwertiger Funktionen, die einen Hilbertraum bilden, werden hier vektorwertige Abbildungen betrachtet, die einen Pontrjaginraum bilden. Diese Verallgemeinerung ist von L. de Branges [3] in einem Spezialfall durchgeführt; er betrachtet analytische Vektorfunktionen, die einen Hilbertraum bilden.

0.2. Im ersten Kapitel sind Grundbegriffe der Theorie der Pontrjaginräume gesammelt. Für weitere Resultate sei auf [4], [5] und [7] verwiesen.

Die Kapitel 2 und 3 enthalten die Theorie der Pontrjaginräume mit einem reproduzierenden Kern. Dabei sind nur die wichtigsten und in den Anwendungen vorkommenden Resultate aus [1] und [11] für den verallgemeinerten Fall übertragen.

Als erste Anwendung betrachten wir in Kapitel $4 *$-Halbgrupppen und Gruppen sowie ihre Darstellungen in einem Pontrjaginraum.

Als zweite Anwendung werden in Kapitel 5 verschiedene verallgemeinerte Resolventen von $\pi$-selbstadjungierten und $\pi$-hermiteschen Operatoren charakterisiert.

\section{Pontrjaginräume}

1.1. Geometrie und Topologie. Es sei $\mathfrak{\Omega}$ ein komplexer linearer Raum, in dem eine hermitesche Form $[\cdot \mid \cdot]: \mathfrak{L} \times \mathfrak{L} \rightarrow \boldsymbol{C}$ gegeben ist.

Ein Element $f$ von $\mathfrak{Q}$ wird nichtnegativ (bzw. positiv, neutral, usw.) genannt, wenn $[f \mid f] \geq 0$ (bzw. $[f \mid f]>0,[f \mid f]=0$, usw.) gilt. Entsprechende Definitionen gelten auch für Teilräume von $\mathfrak{\Omega}$.

Ein Paar von Vektoren $f, g$ (von Teilmengen $\mathfrak{M}, \mathfrak{N}$ ) des Raumes $\Omega$ heißt $\pi$-orthogonal (bezüglich $[\cdot \mid \cdot])$, in Zeichen $f \perp g(\mathfrak{M} \perp \mathfrak{R})$, wenn $[f \mid g]=0 \quad([f \mid g]=0$ für alle $f \in \mathfrak{M}, g \in \mathfrak{N})$ ist. Für jede Teilmenge $\mathfrak{M}$ von $\mathfrak{Q}$ ist die Menge derjenigen Vektoren $f$, die zu $\mathfrak{M} \pi$-ortho- 
gonal sind, ein Teilraum von $\mathfrak{Q}$; man nennt ihn das $\pi$-orthogonale Komplement $\mathfrak{M} \perp$ von $\mathfrak{M}$. Eine Teilmenge $\mathfrak{M}$ heißt total, wenn $\mathfrak{M}^{\perp}=\{0\}$.

Falls in einem Teilraum $\mathfrak{M}$ ein Vektor $f \neq 0$ existiert, der auch zu $\mathfrak{M}^{\perp}$ gehört, heißt $\mathfrak{M}$ (oder die Form $[\cdot \mid \cdot]$ auf $\mathfrak{M}$ ) entartet.

Der Raum $\mathfrak{Q}$ heißt ein $\pi_{*}$-Lineal (wobei $*$ eine natürliche Zahl ist), falls die Form $[\cdot \mid \cdot]$ auf $\mathfrak{Q}$ genau $\varkappa$ negative Quadrate hat; dies bedeutet, daß alle negativen Teilräume von $\mathfrak{R}$ die Dimension $\leq x$ haben und mindestens ein solcher Teilraum der Dimension $*$ existiert. In diesem Fall nennt man die Form $[\cdot \mid \cdot]$ ein $\pi$-Skalarprodukt. Ist das $\pi_{x}$-Lineal $\mathfrak{L}$ nicht entartet, so heißt $\mathfrak{L}$ ein Praepontrjaginraum (mit $*$ negativen Quadraten).

In dem Praepontrjaginraum $\mathfrak{Q}$ erklären wir zwei Konvergenzbegriffe folgendermaßen: Eine Folge $\left(f_{n}\right)$ in $\mathfrak{L}$ konvergiert gegen $f \in \mathfrak{Q}, f_{n} \rightarrow f$, falls die Relationen 1) $\left.\left[f_{n} \mid f_{n}\right] \rightarrow[f \mid f], 2\right)\left[f_{n} \mid g\right] \rightarrow[f \mid g]$ für alle $g$ aus $\mathfrak{L}$, gelten. Wenn nur die zweite Bedingung erfüllt ist, sagen wir, daß $\left(f_{n}\right)$ schwach gegen $f$ konvergiert, $f_{n} \rightarrow f$.

Der Praepontrjaginraum $\&$ gestattet eine (i. allg. nicht eindeutige) Zerlegung als direkte Summe zweier $\pi$-orthogonaler Teilräume:

$$
\mathfrak{L}=\mathfrak{\Omega}_{+}+\mathfrak{Q}_{-} ;
$$

wobei $\mathfrak{L}_{+}$(bzw. $\mathfrak{\Omega}_{-}$) positiv (bzw. negativ) ist und $\operatorname{dim} \mathfrak{\Omega}_{-}=*$ gilt.

Die Zerlegung (1.1) erzeugt in $\mathfrak{\Omega}$ eine nichtentartete positive hermitesche Form, d.h. ein gewöhnliches Skalarprodukt, $(\cdot \mid \cdot)$ :

$$
(f \mid g):=\left[f_{+} \mid g_{+}\right]-\left[f_{-} \mid g_{-}\right]
$$

dabei sind $f_{+}, g_{+}$(bzw. $f_{-}, g_{-}$) die Komponenten der Elemente $f, g$ von $\mathfrak{Z}$ in $\mathfrak{Z}_{+}$(bzw. $\mathfrak{L}_{-}$) bezüglich der Zerlegung (1.1). Der Raum $\mathfrak{L}$ versehen mit der Form (1.2) ist ein Praehilbertraum und

$$
|[f \mid g]| \leq\|f\|_{\|} \| \quad \text { für alle } f, g \in \Omega,
$$

wobei $\|f\|:=(f \mid f)^{1 / 2}$ ist.

Wenn in einer Zerlegung (1.1) des Praepontrjaginraumes $\Omega$ der Teilraum $\mathfrak{\Omega}_{+}$vollständig (d.h. ein Hilbertraum) bezüglich des $\pi$-Skalarproduktes $[\cdot \mid \cdot]$ ist, so gilt dasselbe für jede solche Zerlegung; in diesem Fall heißt $\mathfrak{Q}$ Pontrjaginraum (mit * negativen Quadiaten) oder $\tau_{\text {- }}$-Raum, den wir im folgenden häufig mit $\Pi_{\%}$ bezeichnen. Dann ist $\Pi_{\varkappa}:=\Omega$ ein Hilbertraum bezüglich des Skalarproduktes $(\cdot \mid \cdot)$ aus (1.2).

Bekanntlich läßt sich jeder Praepontrjaginraum $\&$ zu einem Pontrjaginraum $\hat{\Omega} \quad$ vervollständigen. ${ }^{1}$ )

1) Man beachte, daß aus $f_{n} \rightarrow f$ in $\mathfrak{L}$ i. allg. nicht $f_{n} \Rightarrow f$ in $\hat{\mathfrak{Q}}$ folgt. 
Wir bemerken, daß in $\Pi_{\varkappa}$ die Relation $f_{n} \rightarrow f$ (bzw. $f_{n} \rightarrow f$ ) genau dann gilt, wenn $f_{n}$ gegen $f$ im Sinne der oben eingeführten Norm $\|\cdot\|$ konvergiert (bzw. schwach konvergiert). Im folgenden beziehen sich die topologischen Begriffe auf diese Normtopologie.

1.2. Operatoren in $\left.\Pi_{\varkappa} \cdot{ }^{2}\right)$ Es sei jetzt $\Pi_{\varkappa}$ ein Pontrjaginraum und $T$ ein dicht definierter Operator in $\Pi_{\varkappa}$. Wir erklären den $\pi$-adjungierten Operator $T^{+}$von $T$ folgendermaßen: $\mathfrak{D}\left(T^{+}\right)$ist die Menge aller Elemente $g \in \Pi_{x}$, zu denen ein Element $h \in I_{\varkappa}$ existiert mit $[T f \mid g]=[f \mid h]$ für alle $f \in \mathscr{D}(T)$; und es sei $T+g:=h$.

Wir bemerken, daß diese Definition für einen in $\Pi_{\%}$ dicht definierten Operator mit Werten in einem anderen Pontrjaginraum $\Pi_{\varkappa^{\prime}}^{\prime}$ verallgemeinert werden kann. Insbesondere gilt für $T \in \mathcal{B}\left(\Pi_{\varkappa^{\prime}} ; \Pi_{\varkappa^{\prime}}^{\prime}\right)$ auch $T+\epsilon B\left(\Pi_{x^{\prime}}^{\prime} ; \Pi_{x}\right)$.

Einen in $\Pi_{\varkappa}$ dicht definierten Operator $T$ nennen wir $\pi$-hermitesch, wenn $T \subset T^{+}$, d.h. $[T f \mid g]=[f \mid T g]$ für alle $f, g \in \mathfrak{D}(T)$, und $\pi^{-}$ selbstadjungiert, wenn $T=T^{+}$gilt. Jeder $\pi$-hermitesche Operator $T$ gestattet eine Abschließung, die mit $T^{++}:=\left(T^{+}\right)^{+}$zusammenfällt.

Ein Operator $P \in \mathscr{B}\left(\Pi_{x}\right)$ mit $P^{+}=P^{2}=I \quad(:=$ der identische Operator auf $\Pi_{\varkappa}$ ) heißt $\pi$-orthogonaler Projektor.

Sei jetzt $T$ ein Operator in $\Pi_{\kappa}$, der nicht notwendig dicht definiert ist. Wir nennen $T \pi$-kontrahierend, falls $[T f \mid T f] \leq[f \mid f]$ für alle $f$ aus $\mathfrak{D}(T)$ gilt; steht in dieser Beziehung stets das Gleichheitszeichen, so ist $T \pi$-isometrisch. Ein $\pi$-isometrischer Operator $T$ heißt $\pi$-unitär, wenn $\mathfrak{D}(T)=\mathfrak{R}(T)=\Pi$. ist. Ein Operator $T$ ist genau dann $\pi$-unitär, wenn $T T^{+}=T+T=I$ gilt.

Jeder dicht definierte $\pi$-isometrische Operator $T$ mit einer dichten Wertemenge ist stetig und also durch Stetigkeit $\mathrm{zu}$ einem $\pi$-unitären Operator auf $\Pi_{x}$ fortsetzbar.

1.3. (S5-Kern. Ist $\mathfrak{S}$ ein $\pi_{\varkappa}$-Raum und $\mathfrak{X}$ eine nichtleere Menge, so nennen wir eine Abbildung $K: \mathfrak{X} \times \mathfrak{X} \rightarrow \mathscr{B}(\mathbb{S})$ einen $\mathfrak{S}$-Kern (auf $\mathfrak{X}$ ).

Definition 1.1. Der $\mathfrak{S}-K e r n ~ K$ hat auf $\mathfrak{X}$ genau * negative Quadrate, wenn folgende zwei Bedingungen erfüllt sind:

2) Wir benutzen die folgenden Bezeichnungen: $\mathcal{F}(\mathfrak{X} ; \mathfrak{Y}):=$ die Menge aller Abbildungen von $\mathfrak{X}$ in $\mathfrak{Y} ; \mathfrak{Y}(\mathfrak{X} ; \mathfrak{Y}):=$ die Menge aller Operatoren (d. h. lineare Abbildungen) aus $\mathcal{F}(\mathfrak{X} ; \mathfrak{Y})$, die stetig sind; $\mathscr{Z}(\mathfrak{X}):=\mathscr{B}(\mathfrak{X} ; \mathfrak{X}) . \mathrm{Zu}$ einem Operator $T$ wird mit $\mathfrak{D}(T), \mathfrak{R}(T)$ bzw. $\mathfrak{N}(T)$ die Definitions-, Werte- bzw. Nullmenge von $T$ bezeichnet. 
1) $K(x, y)=K(y, x)^{+}$für alle $x, y \in \mathfrak{X}$.

2) Für jede natürliche Zahl $n \geq 1$ und für beliebige endliche Folgen $\left(u_{i}\right)_{1 \leqq i \leqq n}$ in (S) und $\left(x_{i}\right)_{1 \leqq i \leqq n}$ in $\mathfrak{X}$ hat die (nach 1) hermitesche) Matrix

$$
\left(\left[K\left(x_{i}, x_{j}\right) u_{j} \mid u_{i}\right]\right)_{1 \leqq i, j \leqq n}
$$

höchstens $\varkappa$ negative Eigenwerte und für mindestens eine solche Wahl von $n$ und $\left(u_{i}\right),\left(x_{i}\right)$ genau $x$ negative Eigenwerte.

Ist insbesondere $\varkappa=0$, so heißt der (5)-Kern $K$ positiv definit.

Wenn wir zum Beispiel einen $\pi_{\%}$-Raum $\Pi_{\%}$ mit dem $\pi$-Skalarprodukt $[\cdot \mid \cdot]_{\text {r }}$ betrachten sowie als $\mathscr{C S}$ die komplexe Ebene $C$ und als $\mathfrak{X}$ den Raum $\Pi_{x}$ wählen, so hat der $C$-Kern $[\cdot \mid \cdot]_{x}: \Pi_{x} \times \Pi_{x} \rightarrow C$ auf $\Pi_{x}$ genau $\approx$ negative Quadrate. Dabei ist also $C$ ein $\pi_{0}$-Raum, d.h. ein Hilbertraum, mit dem Skalarprodukt $[\lambda \mid \mu]:=\lambda \bar{\mu}$, und die Menge $S_{(}(C)$ ist natürlicherweise mit $C$ identifiziert.

\section{Reproduzierende Kerne}

2.1. Definition und Eindeutigkeit. In diesem Kapitel sei (5) ein $\pi_{\varkappa}$-Raum mit dem $\pi$-Skalarprodukt $[\cdot \mid \cdot]$ und $\mathfrak{X}$ eine nichtleere Menge.

Definition 2.1. Es sei $\mathfrak{E} \subset \mathcal{J}(\mathfrak{X} ;(\mathfrak{S})$ ein $\pi$-Raum. Ein (5)-Kern $K$ wird einen reproduzierender Kern für 拨 genannt, falls folgende zwei Bedingungen erfüllt sind:

1) Für alle $y \in \mathfrak{X}, u \in \mathfrak{S}$ gehört die durch $x \mapsto K(x, y) u$ erklärte Abbildung $K(\cdot, y) u: \mathfrak{X} \rightarrow \mathfrak{b} \quad z u$ [E.

2) Für alle $y \in \mathfrak{X}, u \in \mathfrak{S}, f \in \mathbb{E}$ hat $K$ die reproduzierende Eigenschaft:

$$
[f(y) \mid u]=[f \mid K(\cdot, y) u] .
$$

Wir bemerken, daß diese Definition z. B. auch dann sinnvoll bleibt, wenn E nur ein Praepontrjaginraum ist.

Falls man als (b) die komplexe Ebene $C$ und als $(5$ ein Hilbertraum mit Skalarprodukt $(\cdot \mid \cdot)$ wählt sowie $5(C)$ mit $C$ identifiziert, erhält man die klassische Definition des Hilbertraumes mit einem reproduzierenden Kern. In diesem Fall hat die reproduzierende Eigenschaft (2.1) die Form

$$
f(y)=(f \mid K(\cdot, y)) .
$$

Um die Eindeutigkeit von $K$ und $(5$ zu zeigen beweisen wir zuerst

Lemma 2.2. Sei $\mathfrak{E} \subset \mathcal{F}(\mathfrak{X} ;(\mathfrak{S})$ ein Pontrjaginraum mit einem reproduzierenden Kern $K$. Dann ist die Menge

$$
\mathfrak{F}:=\{K(\cdot, y) u \mid y \in \mathfrak{X}, u \in \mathbb{S}\}
$$

total in $\mathfrak{F}, d . h .^{3)}$ a. $1 . \mathrm{H} . \mathfrak{F}=\mathfrak{E}$.

\footnotetext{
3) Wir schreiben: 1.H. := lineare Hülle; a.l.H. := abgeschlossene lineare
} Hülle. 
Beweis. Gehört $f \mathrm{zu} \mathfrak{F}^{\perp}$, so ergibt sich aus (2.1)

$$
0=[f \mid K(\cdot, y) u]=[f(y) \mid u]
$$

für alle $y \in \mathfrak{X}, u \in \mathfrak{B S}$. Demzufolge ist $f=0$, d. h. $\mathfrak{F}$ ist total.

Satz 2.3. Ein Praepontrjaginraum $\mathbb{E} \subset \mathcal{F}(\mathfrak{X} ;(\mathfrak{S})$ hat höchstens einen reproduzierenden Kern.

Falls umgekehrt $K$ ein reproduzierender Kern für zwei Pontrjaginräume ist, fallen diese Räume zusammen.

Beweis. A. Falls \& die reproduzierenden Kerne $K$ und $K^{\prime}$ hat, folgt aus (2.1)

$$
\left[f \mid K(\cdot, y) u-K^{\prime}(\cdot, y) u\right]=[f(y) \mid u]-[f(y) \mid u]=0
$$

für alle $f \in \mathfrak{E}, y \in \mathfrak{X}, u \in \mathbb{S}$. Somit ist $K(\cdot, y) u=K^{\prime}(\cdot, y) u$, also $K=K^{\prime}$.

B. Sei nun $K$ ein gemeinsamer reproduzierender Kern für Pontrjaginräume $\mathbb{F}$ und $\mathfrak{F}^{\prime}$. Dann ist aber nach Lemma $2.2 \quad \mathfrak{F}=$ a. 1. H. $\mathfrak{F}=\mathfrak{F}^{\prime}$, denn $\mathfrak{F}$ hängt nur von $K$ ab.

Der reproduzierende Kern hat die folgende charakteristische Eigenschaft:

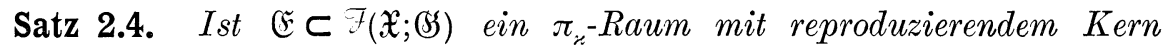
$K$, so ist $K$ ein (SS-Kern mit höchstens $\varkappa$ negativen Quadraten.

Später werden wir zeigen, daß dieser Satz sich umkehren läßt.

Beweis. Sind $x, y \in \mathfrak{X}$ und $u, v \in \mathbb{S}$ beliebig, so erhalten wir aus (2.1)

$$
\begin{aligned}
{[K(x, y) u \mid v] } & =[K(\cdot, y) u \mid K(\cdot, x) v]=\overline{[K(\cdot, x) v \mid K(\cdot, y) u]} \\
& =\overline{[K(y, x) v \mid u]}=[u \mid K(y, x) v]=[K(y, x)+u \mid v] .
\end{aligned}
$$

Somit ist $K(x, y)=K(y, x)^{+}$.

Sei nun $n \geq 1$ beliebig sowie $\left(u_{i}\right)_{1 \leqq i \leqq n}$ und $\left(x_{i}\right)_{1 \leqq i \leqq n}$ beliebige endliche Folgen aus (S) bzw. $\mathfrak{X}$. Dann gilt

$$
\left[K\left(x_{i}, x_{j}\right) u_{j} \mid u_{i}\right]=\left[K\left(\cdot, x_{j}\right) u_{j} \mid K\left(\cdot, x_{i}\right) u_{i}\right] .
$$

Da die Elemente $K\left(\cdot, x_{j}\right) u_{j}$ in $[5$ liegen und das $\pi$-Skalarprodukt $[\cdot \mid \cdot]$ von $[5$ genau $\varkappa$ negative Quadrate hat, folgt daraus, daß $K$ höchstens * negative Quadrate hat.

2.2. Existenz eines reproduzierenden Kerns. Jetzt wird untersucht, wann ein Pontrjaginraum $\mathfrak{E} \subset \mathcal{F}(\mathfrak{X} ;(\mathfrak{S})$ einen reproduzierenden Kern hat.

Satz 2.5. Es sei $\mathfrak{F} \subset \mathcal{F}(\mathfrak{X} ;(S)$ ein Praepontrjaginraum. Für die

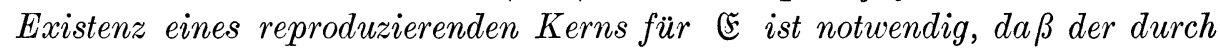

$$
\varphi_{y}(f):=f(y) \quad(f \in \mathbb{E})
$$

für beliebige $y \in \mathfrak{X}$ definierte Operator $\varphi: \mathfrak{F} \rightarrow \mathbb{S S}$ schwach stetig ist. 
A. I. 594

Falls insbesondere E ein Pontrjaginraum ist, ist diese Bedingung auch hinreichend für die Existenz eines reproduzierenden Kerns.

Beweis. A. Sei zuerst (5 ein Praepontrjaginraum mit reproduzierendem Kern $K$. Dann ist $\varphi_{y}$ schwach stetig, weil aus (2.1) und (1.3)

$$
\left|\left[\varphi_{y}(f) \mid u\right]\right|=|[f(y) \mid u]|=|[f \mid K(\cdot, y) u]| \leq\|f\|\|K(\cdot, y) u\| .
$$

folgt.

B. Sei nun $₹$ ein Pontrjaginraum. Da der Darstellungssatz von F. Riesz auch für das $\pi$-Skalarprodukt gültig ist (siehe [4], Lemma 2.1) und nach Voraussetzung $\left[\varphi_{y}(\cdot) \mid u\right]$ in $\mathscr{B}(\mathfrak{E} ; C)$ liegt, existiert für jedes $y \in \mathfrak{X}$ und $u \in \mathbb{S}$ ein eindeutiges Element $K_{y}(u) \in \mathbb{E}$, so daß die Beziehung

$$
\left[\varphi_{y}(f) \mid u\right]=\left[f \mid K_{y}(u)\right]
$$

für alle $f \in\left[\right.$ besteht. Man verifiziert leicht, daß dann $K_{y}$ in $\mathscr{S}$ (SS; (5) liegt. Demzufolge gibt es zu $K_{y}$ einen $\pi$-adjungierten Operator $K_{y}^{+} \epsilon$ B(C; $;$ S ) derart, daß

$$
\left[K_{y}^{+} f \mid u\right]=\left[f \mid K_{y} u\right]
$$

für alle $f \in \mathbb{E}, u \in \mathbb{S}$ gilt.

Wir definieren eine Abbildung $K: \mathfrak{X} \times \mathfrak{X} \rightarrow \mathcal{Y}(\mathbb{S})$ durch

$$
K(x, y):=K_{x}^{+} K_{y}
$$

für alle $x, y \in \mathfrak{X}$ und zeigen, daß $K$ ein reproduzierender Kern für \& ist.

Zuerst folgt aus (2.5), (2.4) und (2.3) für alle $x \in \mathfrak{X}, f \in \mathbb{E}, u \in \mathfrak{S}$ die Relation

$$
\left[K_{x}^{+} f \mid u\right]=\left[f \mid K_{x} u\right]=\left[\varphi_{x}(f) \mid u\right]=[f(x) \mid u],
$$

also ist $K_{x}^{+} f=f(x)$. Damit erhalten wir

$$
K(x, y) u=K_{x}^{+}\left(K_{y} u\right)=\left(K_{y} u\right)(x) .
$$

Demzufolge liegt $K(\cdot, y) u=K_{y} u$ in $\mathfrak{E}$ für alle $y \in \mathfrak{X}, u \in \mathbb{G S}$.

Der $\mathscr{S}$-Kern $K$ hat auch die reproduzierende Eigenschaft (2.1), denn nach (2.6) und (2.7) gilt

$$
[f(y) \mid u]=\left[K_{y}^{+} f \mid u\right]=[f \mid K(\cdot, y) u] .
$$

Somit ist der Satz bewiesen.

Folgerung 2.6. Jeder abgeschlossene nichtentartete Teilraum eines Pontrjaginraumes \& mit dem reproduzierenden Kern hat auch einen reproduzierenden Kern.

Sei nämlich $\mathfrak{E}_{1} \subset \mathbb{E}$ ein solcher Teilraum. Nach Satz 2.5 ist der in (2.3) erklärte Operator $\varphi_{y}: \mathbb{E} \rightarrow$ (5) schwach stetig und deshalb ist auch 
die Einschränkung $\left.\varphi_{y}\right|_{\mathfrak{E}_{1}}$ schwach stetig. Da $\mathbb{E}_{1}$ ein Pontrjaginraum ist, folgt aus Satz 2.5 die Behauptung.

In diesem Fall können wir den $\pi$-orthogonalen Projektor auf $\mathfrak{F}_{1}$ ausrechnen:

Satz 2.7. Sei $\mathbb{E} \subset \mathcal{F}(\mathfrak{X} ; G)$ ein Pontrjaginraum und $\mathfrak{F}_{1} \subset \mathbb{E}$ ein nichtentarteter, abgeschlossener Teilraum mit reproduzierendem Kern $K_{1}$. Dann ist der durch

$$
\left[\left(P_{1} f\right)(y) \mid u\right]=\left[f \mid K_{1}(\cdot, y) u\right]
$$

für alle $f \in \mathbb{E}, y \in \mathfrak{X}, u \in \mathbb{S}$ gegebene Operator $P_{\mathbf{1}}$ der $\pi$-orthogonale Projektor von $\mathbb{E}$ auf $\mathbb{F}_{1}$.

Beweis. Zuerst bemerken wir, daß Relation (2.8) den Operator $P_{1}$ eindeutig bestimmt.

Nach Vorraussetzung ist $\mathbb{E}$ in der Form $\mathbb{E}=\mathbb{E}_{1}+\mathfrak{F}_{1}^{\perp}$ darstellbar. Sei $f=f_{1}+f_{1}^{\perp}$ die entsprechende Zerlegung von $f$ aus $\&$. Dann gilt für alle $y \in \mathfrak{X}, u \in \mathbb{S}$

$$
\left[\left(P_{1} f\right)(y) \mid u\right]=\left[f \mid K_{1}(\cdot, y) u\right]=\left[f_{1} \mid K_{1}(\cdot, y) u\right]=\left[f_{1}(y) \mid u\right],
$$

denn $f_{1}^{\perp} \perp K_{1}(\cdot, y) u$. Also ist $P_{1} f=f_{1}$.

Folgerung 2.8. Seien $\mathfrak{E} \subset \mathcal{F}(X ; \mathbb{B S})$ ein Pontrjaginraum und $\mathfrak{E}_{i}$, $i=1,2$, zwei abgeschlossene nichtentartete Teilräume mit den reproduzieren-

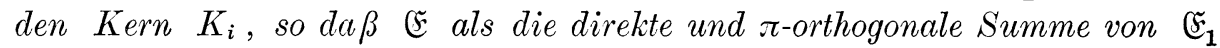
und $\mathfrak{F}_{2}$ darstellbar ist. Dann hat $\mathfrak{E}$ den reproduzierenden Kern $K:=$ $K_{1}+K_{2}$.

Beweis. Es ist klar, daß $K(\cdot, y) u$ für alle $y \in \mathfrak{X}, u \in \mathbb{S}$ zu $\mathbb{E}$ gehört.

Der Kern $K$ hat aber auch die reproduzierende Eigenschaft, denn aus Satz 2.7 folgt

$$
\begin{aligned}
{[f(y) \mid u] } & =\left[\left(\left(P_{1}+P_{2}\right) f\right)(y) \mid u\right] \\
& =\left[f \mid K_{1}(\cdot, y) u\right]+\left[f \mid K_{2}(\cdot, y) u\right]=[f \mid K(\cdot, y) u]
\end{aligned}
$$

für alle $f \in \mathfrak{F}, y \in \mathfrak{X}, u \in \mathfrak{S}$; dabei bezeichnet $P_{i}$ den $\pi$-orthogonalen Projektor von $\mathbb{E}$ auf $\mathfrak{E}_{i}, i=1,2$.

2.3. Konvergenz und Separabilität. Zuerst werden wir untersuchen, wie die Konvergenzen in $\mathbb{E}$ und (5) voneinander abhängen.

Satz 2.9. $\quad$ Es sei $\mathfrak{E} \subset \mathcal{F}(\mathfrak{X} ;(S)$ ein Praepontrjaginraum mit dew reproduzierenden Kern $K$. Wenn eine Folge $\left(f_{n}\right)$ in $[5$ schwach gegen $f \in \mathfrak{E}$ konvergiert, so konvergiert die Folge $\left(f_{n}(x)\right)$ für jedes $x \in \mathfrak{X}$ schwach gegen $f(x)$.

Aus der reproduzierenden Eigenschaft folgt nämlich für alle $x \in \mathfrak{X}$, $u \in \mathbb{B S}$ 


$$
\left[f_{n}(x) \mid u\right]=\left[f_{n} \mid K(\cdot, x) u\right] \rightarrow[f \mid K(\cdot, x) u]=[f(x) \mid u],
$$

d.h. $f_{n}(x) \rightarrow f(x)$ für jedes $x \in \mathfrak{X}$.

Dieser Satz läßt sich partiell umkehren:

Satz 2.10. Es sei $\mathfrak{E} \subset \mathcal{F}(\mathfrak{X} ;(\mathfrak{S})$ ein Pontrjaginraum mit reproduzierendem Kern. Wenn eine beschränkte Folge $\left(f_{n}\right)$ in $\mathfrak{E}_{\mathfrak{E}}$ so gegeben ist, da $\beta$ $f_{n}(x) \rightarrow f(x)$ für alle $x \in \mathfrak{X}$ mit $f \in \mathfrak{F}$ gilt, so konvergiert die Folge $\left(f_{n}\right)$ in $\&$ schwach gegen $f$.

In der Tat, für alle $x \in \mathfrak{X}, u \in \mathbb{S}$ gilt

$$
\left[f_{n} \mid K(\cdot, x) u\right]=\left[f_{n}(x) \mid u\right] \rightarrow[f(x) \mid u]=[f \mid K(\cdot, x) u] .
$$

Nach Lemma 2.2. ist aber die Menge $\{K(\cdot, x) u \mid x \in \mathfrak{X}, u \in \mathbb{S}\}$ total in $\mathbb{F}$. Da die Folge $\left(f_{n}\right)$ beschränkt ist, folgt daraus die Behauptung.

Um die Separabilität von \& zu untersuchen setzen wir jetzt voraus, daß $\mathfrak{X}$ ein separabler topologischer Raum ist.

Satz 2.11. Es sei $\mathfrak{E} \subset \mathcal{F}(\mathfrak{X} ;(\mathfrak{S})$ ein Pontrjaginraum mit reproduzierendem Kern $K$, und seien die Elemente von [5 schwach stetig. Ist (5) separabel, so ist auch [E separabel.

Beweis. Nach Voraussetzung gibt es abzählbare Mengen $\mathfrak{Y}$ in $\mathfrak{X}$ und $\mathfrak{H}$ in $\mathscr{S}$ so, daß $\mathfrak{Y}$ bzw. $\mathfrak{H}$ dicht in $\mathfrak{X}$ bzw. in $(\mathfrak{S}$ liegt.

Wir beweisen, daß die abzählbare Menge

$$
\mathfrak{F}_{\mathfrak{Y}, \mathfrak{S}}:=\{K(\cdot, y) u \mid y \in \mathfrak{Y}, u \in \mathfrak{S}\}
$$

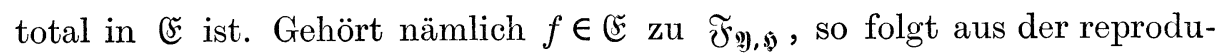
zierenden Eigenschaft

$$
0=[f \mid K(\cdot, y) u]=[f(y) \mid u]
$$

für alle $y \in \mathfrak{Y}, u \in \mathfrak{H}$. Somit ist $\left.f\right|_{\mathfrak{Y}}=0$. Andererseits ist $f$ stetig und (S) ein Hausdorffscher Raum bezüglich der schwachen Topologie. Demzufolge gilt notwendig $f=0$.

Also ist (E) separabel.

\section{Vervollständigung der Pontrjaginräume und induzierte Räume}

3.1. Vervollständigung. Es sei wieder $\mathfrak{X}$ eine nichtleere Menge und (5) ein Pontrjaginraum.

Wir wollen jetzt untersuchen, wann ein Praepontrjaginraum $\mathfrak{E} \subset$ $\mathcal{F}(\mathfrak{X} ; \mathfrak{S})$ sich zu einem Pontrjaginraum $\hat{\mathfrak{E}}$ in der Weise vervollständigen läßt, daß auch $\hat{\mathfrak{E}}$ eine Teilmenge von $\mathcal{F}(\mathfrak{X} ;(\mathbb{S})$ ist.

Satz 3.1. Es sei $\mathfrak{E} \subset \mathcal{F}(\mathfrak{X} ; \mathfrak{S})$ ein Praepontrjaginaum. Für die Existenz eines den Raum [5 als eine dichte Untermenge enthaltenden 
Pontrjaginraumes $\hat{\mathfrak{E}} \subset \mathcal{F}(\mathfrak{X} ; \mathfrak{S})$ mit reproduzierendem Kern ist notwendig und hinreichend, da $\beta$ die folgenden Bedingungen erfüllt sind:

1) Für jedes $y \in \mathfrak{X}$ ist der Operator $f \mapsto f(y)$ von $\mathfrak{E}$ in (S) schwach stetig.

2) Für jede Cauchyfolge $\left(f_{n}\right)$ in \& mit det Eigenschaft $f_{n}(y) \rightleftharpoons 0$ für alle $y \in \mathfrak{X}$ gilt $\left[f_{n} \mid f_{n}\right] \rightarrow 0$.

Die Vervollständigung $\hat{\mathfrak{F}}$ ist eindeutig.

Beweis. A. Wir zeigen zuerst die Notwendigkeit. Aus Satz 2.5 folgt, daß 1) erfüllt ist. Sei nun $\left(f_{n}\right)$ eine Cauchyfolge in $\&$ mit $f_{n}(y) \rightarrow 0$ für jedes $y \in \mathfrak{X}$. Dann hat diese Folge einen Grenzwert $f$ in $\hat{\mathfrak{E}}$. Nach Voraussetzung und Satz 2.9 gelten für alle $y \in \mathfrak{X}$ die Beziehungen $f_{n}(y) \rightarrow 0$ und $f_{n}(y) \rightarrow$ $f(y)$, also ist $f=0$. Daraus folgt 2).

B. Um die Hinlänglichkeit zu zeigen stellen wir \& in der Form

$$
\mathfrak{E}=\mathfrak{E}_{+}+\mathfrak{E}_{-} \quad\left(\operatorname{dim} \mathfrak{E}_{-}<\infty\right)
$$

dar und vervollständigen den Praehilbertraum $\mathfrak{F}_{+}$zu einem Hilbertraum, der von Abbildungen gebildet ist.

$\mathrm{Zu}$ diesem Zweck definieren wir $\hat{\mathfrak{E}}_{+}$als den linearen Raum aller Abbildungen $f \in \mathcal{F}(\mathfrak{X} ; \mathfrak{S})$, für die je eine Cauchyfolge $\left(f_{n}\right)$ in $\mathfrak{E}_{+}$mit $f_{n}(x) \rightarrow f(x)$ für jedes $x \in \mathfrak{X}$ existiert.

Man kann eine Norm auf $\mathfrak{E}_{+}$durch

$$
\|f\|^{\wedge}:=\lim _{n \rightarrow \infty}\left\|f_{n}\right\|
$$

erklären, wobei $\left(f_{n}\right)$ eine zu $f \in \widehat{\mathfrak{E}}_{+}$gehörige Cauchyfolge in $\mathfrak{E}_{+}$ist.

Wir zeigen, daß diese Definition sinnvoll ist. Weil $\left(f_{n}\right)$ eine Cauchyfolge ist, existiert der Grenzwert in $\boldsymbol{R}$. Ferner sei $\left(f_{n}^{\prime}\right)$ eine andere Cauchyfolge in $\mathfrak{E}_{+}$mit $f_{n}(x) \rightarrow f(x)$. Dann ist $\left(f_{n}-f_{n}^{\prime}\right)$ eine Cauchyfolge in $\mathfrak{E}_{+}$mit $\left(f_{n}-f_{n}^{\prime}\right)(x) \rightarrow 0$. Aus 2) folgt, daß $\left[f_{n}-f_{n}^{\prime} \mid f_{n}-f_{n}^{\prime}\right] \rightarrow 0$ also $f_{n}-f_{n}^{\prime} \rightarrow 0$ gilt. Demnach erhalten wir

$$
\left|\lim _{n \rightarrow \infty}\left\|f_{n}\right\|-\lim _{n \rightarrow \infty}\left\|f_{n}^{\prime}\right\|\right| \leq \lim _{n \rightarrow \infty}\left\|f_{n}-f_{n}^{\prime}\right\|=0,
$$

d. h. $\lim _{n \rightarrow \infty}\left\|f_{n}\right\|=\lim _{n \rightarrow \infty}\left\|f_{n}^{\prime}\right\|$.

Von den Normeigenschaften von $\|\cdot\|^{\wedge}$ wird hier nur die positive Definitheit verifiziert; die anderen sind offensichtlich. Sei zuerst $f=0$ in $\hat{\mathfrak{F}}_{+}$. Dann gibt es eine Cauchyfolge $\left(f_{n}\right)$ in $\mathfrak{F}_{+}$mit $f_{n}(x) \rightarrow 0$. Aus 2) folgt

$$
\|f\|^{\wedge^{2}}=\lim _{n \rightarrow \infty}\left\|f_{n}\right\|^{2}=\lim _{n \rightarrow \infty}\left[f_{n} \mid f_{n}\right]=0
$$

also $\|\boldsymbol{f}\|^{\wedge}=0$. Sei umgekehrt $\|f\|^{\wedge}=0$ für $f \in \hat{\mathscr{E}}_{+}$und $\left(f_{n}\right)$ eine ent- 
sprechende Cauchyfolge in $\mathfrak{E}_{+}$mit $f_{n}(x) \rightarrow 0$. Nach 1) gibt es eine Konstante $M_{x, u}>0$ mit der Eigenschaft

$$
\left|\left[f_{n}(x) \mid u\right]\right| \leq M_{x, u}\left\|f_{n}\right\|
$$

für alle $x \in \mathfrak{X}, u \in \mathscr{S}$. Demnach gilt

$$
|[f(x) \mid u]|=\lim _{n \rightarrow \infty}\left|\left[f_{n}(x) \mid u\right]\right| \leq M_{x, u} \lim _{n \rightarrow \infty}\left\|f_{n}\right\|=M_{x, u}\|f\|^{\wedge}=0 .
$$

Somit ist $f=0$.

Der Raum $\hat{E}_{+}$ist in $\hat{\mathfrak{F}}_{+}$enthalten, denn für $f \in \mathfrak{E}_{+}$ist die Folge $\left(f_{n}\right)$ mit $f_{n}:=f$ eine Cauchyfolge, die die Eigenschaft $f_{n}(x) \rightarrow f(x)$ hat. Außerdem gilt $\|f\|^{\wedge}=\|f\|$. Der Raum $\hat{\mathfrak{F}}_{+}$liegt sogar dicht in $\hat{\mathfrak{E}}_{+}$. Ist nämlich $f$ in $\hat{\mathfrak{E}}_{+}$, so gibt es eine Cauchyfolge $\left(f_{n}\right)$ in $\mathfrak{E}_{+}$mit $\|f\|^{\wedge}=$ $\lim _{n \rightarrow \infty}\left\|f_{n}\right\|$. Dann hat man

$$
\lim _{n \rightarrow \infty}\left\|f-f_{n}\right\|^{\wedge}=\lim _{n \rightarrow \infty}\left(\lim _{m \rightarrow \infty}\left\|f_{m}-f_{n}\right\|\right)=0 .
$$

Wir beweisen nun, daß $\hat{\mathfrak{E}}_{+}$vollständig ist. Dazu sei $\left(f_{n}\right)$ eine beliebige Cauchyfolge in $\hat{\mathfrak{E}}_{+}$. Dann kann man eine Cauchyfolge $\left(f_{n}^{\prime}\right)$ in $\mathfrak{E}_{+}$so finden, daß

$$
\lim _{n \rightarrow \infty}\left\|f_{n}-f_{n}^{\prime}\right\|^{\wedge}=0
$$

gilt, denn $\mathfrak{E}_{+}$liegt dicht in $\hat{\mathfrak{E}}_{+}$. Nach $(3.1)$ ist $\left(f_{n}(x)\right)$ für jedes $x \in \mathfrak{X}$ eine schwache Cauchyfolge in $\mathbb{S}$. Da (S) auch schwach vollständig ist, existiert für jedes $x \in \mathfrak{X}$ ein Element $f(x) \in \mathbb{S}$ mit $f_{n}(x) \rightarrow f(x)$. Man sieht unmittelbar, daß die Abbildung $f$ zu $\hat{\mathfrak{E}}_{+}$gehört und $f_{n} \rightarrow f$ gilt. In der Tat, aus (3.2) folgt

$\lim _{n \rightarrow \infty}\left\|f_{n}-f\right\|^{\wedge} \leq \lim _{n \rightarrow \infty}\left\|f_{n}-f_{n}^{\prime}\right\|^{\wedge}+\lim _{n \rightarrow \infty} \| f_{n}^{\prime}-f_{\|}^{\wedge}=\lim _{n \rightarrow \infty}\left(\lim _{m \rightarrow \infty}\left\|f_{n}^{\prime}-f_{m}\right\|\right)=0$.

Für $f, g \in \hat{\mathfrak{E}}_{+}$definieren wir

$$
[f \mid g]_{+}:=\lim _{n \rightarrow \infty} \lim _{m \rightarrow \infty}\left[f_{n} \mid g_{m}\right],
$$

wobei $\left(f_{n}\right)$ (bzw. $\left(g_{n}\right)$ ) eine zu $f$ (bzw. $g$ ) gehörende Cauchyfolge in $\mathfrak{E}_{+}$ist. Es ist leicht zu verifizieren, daß die Abbildung $(f, g) \mapsto[f \mid g]_{+}$ von $\hat{\mathfrak{F}}_{+} \times \hat{\mathfrak{E}}_{+}$in $C$ eine wohl definierte hermitesche Form ist, für die

$$
[f \mid f]_{+}^{\hat{n}}=\|f\|^{\wedge_{2}}
$$

gilt. Also ist $\hat{\mathfrak{E}}_{+}$ein Hilbertraum.

Wir bilden die direkte Summe $\hat{F}:=\hat{F}_{+}+\mathfrak{F}_{-}$und setzen 


$$
[f \mid g]^{\wedge}:=\left[f_{+} \mid g_{+}\right]_{+}^{\wedge}+\left[f_{-} \mid g_{-}\right]
$$

für $f, g \in \widehat{\mathfrak{E}}$, wobei $f_{+}, g_{+}$(bzw. $f_{-}, g_{-}$) die Komponenten der Elemente

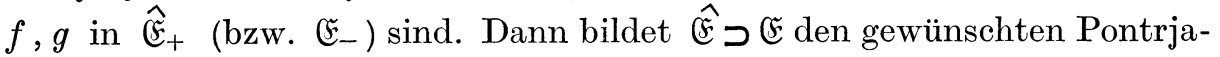
ginraum, in dem E dicht liegt. Aus (3.1) und Satz 2.5 schließt man weiter, daß der Raum $\hat{E}$ einen reproduzierenden Kern hat.

C. Um die Eindeutigkeit der Vervollständigung von \& zu beweisen, nehmen wir die Existenz einer anderen solchen Vervollständigung $\tilde{\mathfrak{E}}$ von $\mathfrak{E}$ an. Gehört ein Element $f$ zu $\tilde{E}$, so ist $f$ der Grenzwert einer Cauchyfolge $\left(f_{n}\right)$ in $\mathfrak{E}$. Nach Satz 2.9 gilt dann $f_{n}(x) \rightarrow f(x)$, also liegt $f$ in $\hat{\mathfrak{E}}$. Falls umgekehrt $f$ in $\hat{\mathfrak{E}}$ ist, gibt es eine Cauchyfolge $\left(f_{n}\right)$ in $\mathfrak{E}$ mit $f_{n}(x) \rightarrow f(x)$. Dann hat diese Folge einen Grenzwert $f^{\prime}$ in $\tilde{\mathbb{E}}$. Aus Satz 2.9 folgt, daß $f^{\prime}=f$ ist. Somit gilt $\hat{\mathfrak{E}}=\tilde{\mathfrak{E}}$ und die $\pi$-Skalarprodukte von $\hat{\mathfrak{E}}$ und $\tilde{E}$ müssen auch übereinstimmen, da $\mathfrak{E}$ dicht sowohl in $\hat{E}$ als auch in $\tilde{E}$ liegt.

Folgerung 3.2. Es sei $\mathfrak{E} \subset \mathcal{F}(\mathfrak{X} ; \mathfrak{S})$ ein Praepontrjaginraum mit reproduzierendem Kern $K$. Ein E umfassender Pontrjaginraum $\hat{\mathbb{E}}$ mit reproduzierendem Kern $K$ existiert genau dann, wenn Bedingung 2) aus Satz 3.1 erfüllt ist.

Der Beweis der Notwendigkeit von 2) verläuft genau so wie Teil A des Beweises von Satz 3.1.

Umgekehrt folgt aus Satz 2.5, daß Bedingung 1) von Satz 3.1 erfüllt ist. Demnach existiert ein $\mathfrak{E}$ umfassender Pontrjaginraum $\hat{\mathfrak{E}}$ mit reproduzierendem Kern $K^{\prime}$. Da $\mathfrak{E}$ dicht in $\hat{\mathfrak{F}}$ liegt, müssen die Kerne $K$ und $K^{\prime}$ übereinstimmen.

Es sei noch vermerkt, daß die Räume $\mathfrak{E}$ und $\hat{\mathfrak{E}}$ in Satz 3.1 und Folgerung 3.2 die gleiche Anzahl negativer Quadrate haben.

3.2. Induzierte Räume. Es sei wieder $\mathfrak{X}$ eine nichtleere Menge und (5) ein Pontrjaginraum. Wir zeigen nun, daß Satz 2.4 sich folgendermaßen umkehren läßt:

Satz 3.3. Ist $K$ ein (5)-Kern auf $\mathfrak{X}$, der $\approx$ negative Quadrate hat, dann existiert ein $\pi_{*}$-Raum $\mathfrak{E} \subset \mathcal{F}(\mathfrak{X} ;(\mathfrak{S})$ mit reproduzierendem Kern $K$. Genauer, der Raum (E) ist die Vervollständigung der Praepontrjaginraumes $\mathfrak{L}$, der alle Abbildungen $f: \mathfrak{X} \rightarrow \mathfrak{B S}$ der Form

$$
f=\sum_{i=1}^{m} K\left(\cdot, y_{i}\right) u_{i}
$$

mit $y_{i} \in \mathfrak{X}$ und $u_{i} \in \mathbb{G S}$ enthält; das $\pi$-Skalarprodukt von $\mathfrak{Q}$ ist durch 


$$
[f \mid g]:=\sum_{i=1}^{m} \sum_{j=1}^{n}\left[K\left(z_{j}, y_{i}\right) u_{i} \mid v_{j}\right]
$$

gegeben für $f$ und

$$
g:=\sum_{j=1}^{n} K\left(\cdot, z_{j}\right) v_{j}
$$

Beweis. Es ist klar, daß die oben definierte Menge $\mathfrak{Q}$ einen komplexen linearen Raum bildet. Die für $f, g \in \mathfrak{\Omega}$ gültigen Beziehungen

$$
[f \mid g]=\sum_{j=1}^{n}\left[f\left(z_{j}\right) \mid v_{j}\right]=\sum_{i=1}^{m}\left[u_{i} \mid g\left(y_{i}\right)\right]
$$

zeigen, daß $[f \mid g]$ unabhängig von der Darstellung von $f$ bzw. $g$ ist. Die Abbildung $[\cdot \mid \cdot]: \mathfrak{L} \times \mathfrak{L} \rightarrow \boldsymbol{C}$, die offensichtlich eine hermitesche Form ist, hat nach Voraussetzung genau $\approx$ negative Quadrate auf $\Omega$. Sie ist auch nichtentartet, denn aus $[f \mid g]=0$ für alle $g \in \mathfrak{\Omega}$ folgt nach $(3.6)$

$$
0=[f \mid K(\cdot, y) u]=[f(y) \mid u]
$$

für alle $y \in \mathfrak{X}$ und $u \in \mathfrak{S}$, also $f=0$.

Somit bildet $\mathbb{Q}$ einen Praepontrjaginraum, der nach (3.6) den reproduzierenden Kern $K$ hat. Wir zeigen noch, daß Bedingung 2) von Satz 3.1 erfüllt ist. Dazu sei $\left(f_{k}\right)$ eine Cauchyfolge in $\mathfrak{L}$ mit der Eigenschaft $f_{k}(y) \rightarrow 0$ für alle $y \in \mathfrak{X}$. Mit Hilfe von (3.6) erhalten wir

$$
\left[f_{k} \mid g\right]=\sum_{j=1}^{n}\left[f_{k}\left(z_{j}\right) \mid v_{j}\right] \rightarrow 0
$$

für alle $g \in \mathfrak{\Omega}$. Als Cauchyfolge ist $\left(f_{k}\right)$ beschränkt, d.h. $\left\|f_{k}\right\| \leq M$, und somit ergibt sich weiter

$$
\left|\left[f_{k} \mid f_{k}\right]\right| \leq\left|\left[f_{l} \mid f_{k}\right]\right|+\left|\left[f_{k}-f_{l} \mid f_{k}\right] \leq\left[f_{l} \mid f_{k}\right]:+M\right| \mid f_{k}-f_{l} \| .
$$

Aus (3.7) und dieser Beziehung folgt die Relation $\left[f_{k}: f_{k}\right] \rightarrow 0$.

Nach Folgerung 3.2 ist Satz 3.3 damit bewiesen.

Wir bemerken noch, daß Satz 3.3 in [7] - [10] für verschiedene spezielle Kerne sogar im Pontrjaginraum bewiesen ist.

\section{4. $\pi$-unitäre Darstellungen von Gruppen}

4.1. Definitionen. Als erste Anwendung der obigen Resultate untersuchen wir Darstellungen von Gruppen in einem Pontrjaginraum. Zuerst geben wir einige Definitionen. 
Eine Menge $\mathfrak{X}$ heißt eine *-Halbgruppe, wenn in $\mathfrak{X}$ eine assoziative Komposition $(x, y) \mapsto x y$ und ein Einselement $e$ sowie eine Involution $x \mapsto x^{*}$ gegeben sind, d. h. für alle $x, y, z \in \mathfrak{X}$ gelten die folgenden Relationen

1) $x(y z)=(x y) z=: x y z$

2) $x e=e x=x$;

3) $e^{*}=e, x^{* *}=x$ und $(x y)^{*}=y^{*} x^{*}$.

Offenbar ist jede Gruppe eine *-Halbgruppe, wenn man nur $x^{*}:=x^{-1}$ setzt.

Unter einer Darstellung der *-Halbgruppe $\mathfrak{X}$ in einem Pontrjaginraum (5) versteht man eine Abbildung $U: \mathfrak{X} \rightarrow \mathscr{B}($ (S) mit den Eigenschaften

$$
U(e)=I, \quad U(x y)=U(x) U(y), \quad U\left(x^{*}\right)=U(x)^{+}
$$

für alle $x, y \in \mathfrak{X}$.

Die Darstellung $U$ einer *-Halbgruppe $\mathfrak{X}$ in (S) heißt bis auf eine Isomorphie eindeutig bestimmt, falls für jede andere Darstellung $U^{\prime}$ von $\mathfrak{X}$ in $\mathfrak{S S}^{\prime}$ ein $\pi$-isometrischer Operator $V \in \mathcal{B}^{\prime}\left(\mathbb{S}^{\prime} ;(\mathbb{S})\right.$ mit $U^{\prime}(y)=$ $V^{-1} \quad U(y) V$ für alle $y \in \mathfrak{X}$ existiert.

Ist insbesondere $\mathfrak{X}$ eine Gruppe (mit $x^{*}=x^{-1}$ ), so besteht die Dırstellung $U$ aus $\pi$-unitären Operatoren. In diesem Fall spricht man von einer $\pi$-unitären Darstellung.

4.2. Darstellungen von *-Halbgruppen. In diesem Abschnitt sei $\mathfrak{X}$ stets eine *-Halbgruppe und (S) ein $\pi_{\varkappa}$-Raum. Die folgenden zwei Sätze verallgemeinern einige Resultate von B. Sz.-Nagy aus [16].

Satz 4.1. Sei $U$ eine Darstellung der *-Halbgruppe $\mathfrak{X}$ in einem \&s umfassenden $\pi_{- \text {-Raum }}$ \& und $T$ die durch

$$
T(x):=\left.P_{\mathfrak{G}} U(x)\right|_{\mathfrak{G}}
$$

für alle $x \in \mathfrak{X}$ definierte Abbildung, wobei $P_{\mathfrak{E}}$ der $\pi$-orthogonale Projektor von (5) auf (S) ist. Dann gilt:

1) $T(e)=I$ und $T\left(x^{*}\right)=T(x)+$ für alle $x \in \mathfrak{X}$.

2) Der durch

$$
K(x, y):=T\left(x^{*} y\right) \quad(x, y \in \mathfrak{X})
$$

definierte (5)-Kern auf $\mathfrak{X}$ hat höchstens $x$ negative Quadrate.

3) Für alle $z$ und $\left(x_{i}\right)_{1 \leqq i \leqq n}$ in $\mathfrak{X}$ sowie alle $\left(u_{i}\right)_{1 \leqq i \leqq n}$ in (S) gilt

$$
\left|\sum_{i, j=1}^{n}\left[K\left(z x_{i}, z x_{j}\right) u_{j} \mid u_{i}\right]\right| \leq\|U(z)\|^{2}\left\|\sum_{i=1}^{n} U\left(x_{i}\right) u_{i}\right\|^{2} .
$$

In der Tat, aus den Definitionen folgen unmittelbar die Eigenschaften 
1) und 2). Relation (4.3) erhält man aus (4.2), (4.1) und (1.3) folgendermaßen:

mit

$$
\begin{aligned}
\left|\sum_{i, j=1}^{n}\left[K\left(z x_{i}, z x_{j}\right) u_{j} \mid u_{i}\right]\right| & =\left|\sum_{i, j=1}^{n}\left[U\left(x_{i}\right)+U(z)+U(z) U\left(x_{j}\right) u_{j} \mid u_{i}\right]\right| \\
& =|[U(z) f \mid U(z) f]| \leq\|U(z)\|^{2}\|f\|^{2}
\end{aligned}
$$

$$
f:=\sum_{i=1}^{n} U\left(x_{i}\right) u_{i} \in \mathfrak{E} .
$$

Satz 4.1 läßt sich umkehren:

Satz 4.2. Es sei $T: \mathfrak{X} \rightarrow T(\mathbb{S}(5)$ eine Abbildung mit den Eigenschaften:

1) $T(e)=I$ und $T\left(x^{*}\right)=T(x)^{+}$für alle $x \in \mathfrak{X}$.

2) Der $\mathfrak{B S}$-Kern $(x, y) \mapsto K(x, y):=T\left(x^{*} y\right)$ auf $\mathfrak{X}$ hat genau $\approx$ negative Quadrate.

3) Für alle $z$ und $\left(x_{i}\right)_{1 \leqq i \leqq n}$ in $\mathfrak{X}$ sowie alle $\left(u_{i}\right)_{1 \leqq i \leqq n}$ in ஞs gilt

$$
\left|\sum_{i, j=1}^{n}\left[K\left(z x_{i}, z x_{j}\right) u_{j} \mid u_{i}\right]\right| \leq M_{z} \cdot\left|\sum_{i, j=1}^{n}\left[K\left(x_{i}, x_{j}\right) u_{j} \mid u_{i}\right]\right|
$$

mit einer Konstante $M_{z}>0$.

Dann existiert ein (5) umfassender $\pi_{\varkappa}$-Raum $\mathfrak{E} \subset \mathcal{F}(\mathfrak{X} ;(\mathfrak{S})$ mit dem reproduzierenden Kern $K$ und eine Darstellung $U$ von $\mathfrak{X}$ in $\mathfrak{E}$ derart, daß für alle $x \in \mathfrak{X}$ gilt

$$
T(x)=\left.P_{\mathfrak{G}} U(x)\right|_{\mathfrak{G}},
$$

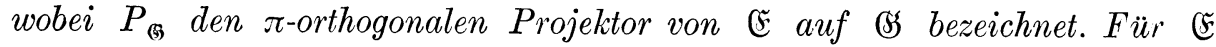
gilt weiter

$$
\mathfrak{F}=\text { a. 1. H. }\{U(y) u \mid y \in \mathfrak{X}, u \in \mathbb{S}\} \text {. }
$$

Wenn ¿E und $U$ diese Bedingungen erfüllen, ist die Darstellung bis auf eine Isomorphie eindeutig bestimmt.

Beweis. Nach Satz 3.3 existiert ein $\pi_{*}$-Raum $\mathfrak{E} \subset \mathcal{F}(\mathfrak{X} ; \mathfrak{S})$ mit reproduzierendem Kern $K$. Wir können (S) in (5) durch $u \mapsto K(\cdot, e) u$ einbetten, den es gilt $[K(\cdot, e) u \mid K(\cdot, e) v]=[u \mid v]$ für alle $u, v \in(5)$.

Bei beliebiger Wahl von $f=\sum_{i=1}^{m} K\left(\cdot, y_{i}\right) u_{i}$ aus der in Satz 3.3 definierten Menge $\mathfrak{Z}$ und von $y \in \mathfrak{X}$ setzen wir

$$
(U(y) f)(x):=\sum_{i=1}^{m} K\left(y^{*} x, y_{i}\right) u_{i}=f\left(y^{*} x\right)
$$

für alle $x \in \mathfrak{X}$. Dann sieht man leicht, daß $U(y)$ ein Operator auf $\&$ ist und 


$$
U(y) f=\sum_{i=1}^{m} K\left(\cdot, y y_{i}\right) u_{i}
$$

gilt.

Für alle $y, z \in \mathfrak{X}$ sowie $f$ und $g$ aus $\mathfrak{L}$ (mit den Darstellungen (3.3) bzw. (3.5)) gelten

$$
\begin{gathered}
U(e) f=\sum_{i=1}^{m} K\left(\cdot, e y_{i}\right) u_{i}=f \\
U(y) U(z) f=\sum_{i=1}^{m} U(y)\left(K\left(\cdot, z y_{i}\right) u_{i}\right)=\sum_{i=1}^{m} K\left(\cdot, y z y_{i}\right) u_{i}=U(y z) f
\end{gathered}
$$

und

$$
\begin{aligned}
{[U(y) f \mid g] } & =\sum_{i=1}^{m} \sum_{j=1}^{n}\left[K\left(z_{j}, y y_{i}\right) u_{i} \mid v_{j}\right]=\sum_{i=1}^{m} \sum_{j=1}^{n}\left[K\left(y^{*} z_{j}, y_{i}\right) u_{i} \mid v_{j}\right] \\
& =\left[f \mid \sum_{j=1}^{n} K\left(\cdot, y^{*} z_{j}\right) v_{j}\right]=\left[f \mid U\left(y^{*}\right) g\right] .
\end{aligned}
$$

Wir zeigen, daß der Operator $U(y)$ in $\mathbb{E}$ stetig ist. Für $f, g \in \mathfrak{Q}$ mit $f \rightarrow 0$ erhalten wir nach $(4.10)$

$$
[U(y) f \mid g]=\left[f \mid U\left(y^{*}\right) g\right] \rightarrow 0
$$

und auf Grund von (4.10), (4.9), (4.4)

$$
\begin{aligned}
|[U(y) f \mid U(y) f]| & =\left|\left[U\left(y^{*} y\right) f \mid f\right]\right|=\left|\sum_{i, j=1}^{m}\left[K\left(y^{*} y y_{i}, y_{j}\right) u_{j} \mid u_{i}\right]\right| \\
& =\left|\sum_{i, j=1}^{m}\left[K\left(y y_{i}, y y_{j}\right) u_{j} \mid u_{i}\right]\right| \leq M_{y}\left|\sum_{i, j=1}^{m}\left[K\left(y_{i}, y_{j}\right) u_{j} \mid u_{i}\right]\right| \\
& =M_{y}|[f \mid f]| \rightarrow 0 .
\end{aligned}
$$

Somit ist $U(y)$ stetig und läßt sich also stetig auf $\&$ fortsetzen, denn $\mathbb{\Omega}$ liegt dicht in $\mathfrak{F}$. Diese Fortsetzung, die wir auch mit $U(y)$ bezeichnen, besitzt offensichtlich die Eigenschaften (4.8)-(4.10) für alle $f, g$ aus $\mathfrak{F}$, d.h. $U: \mathfrak{X} \rightarrow \mathfrak{B}(\mathfrak{E})$ ist eine Darstellung von $\mathfrak{X}$ in $\mathfrak{E}$.

Betrachten wir zwei Elemente $u, v$ von (S) und die zugehörigen Elemente $K(\cdot, e) u, K(\cdot, e) v$ von $\mathfrak{\Omega}$, so gilt

$$
\begin{aligned}
{[U(y) u \mid v] } & =[U(y)(K(\cdot, e) u) \mid K(\cdot, e) v]=[K(\cdot, y) u \mid K(\cdot, e) v] \\
& =\left[K\left(e^{*}, y\right) u \mid v\right]=[T(y) u \mid v]
\end{aligned}
$$

also (4.5).

Gehört $f^{\prime} \mathrm{zu} \mathfrak{Q}$, so hat man nach (4.7)

$$
f=\sum_{i=1}^{m} K\left(\cdot, y_{i}\right) u_{i}=\sum_{i=1}^{m} U\left(y_{i}\right)\left(K(\cdot, e) u_{i}\right)=\sum_{i=1}^{m} U\left(y_{i}\right) u_{i}
$$


Da $\mathfrak{2}$ dicht in $\mathfrak{S}$ liegt, folgt hieraus Relation (4.6).

Um die Eindeutigkeit der Darstellung zu beweisen, nehmen wir an, daß $U^{\prime}$ eine andere Darstellung von $\mathfrak{X}$ in $\mathscr{E}^{\prime}$ mit den in Satz 4.2 genannten Eigenschaften ist. Zwischen den Mengen $\mathbb{L}^{\prime}:=1 . \mathrm{H} .\left\{U^{\prime}(y) u \mid y \in \mathfrak{X}\right.$, $\left.u \in \mathbb{S S}^{\prime}\right\}$ und $\mathfrak{\Omega}=1$. H. $\{U(y) u \mid y \in \mathfrak{X}, u \in \mathfrak{S}\}$ definieren wir eine Abbildung $V$ durch

$$
V f^{\prime}:=V\left(\sum_{i=1}^{m} U^{\prime}\left(y_{i}\right) u_{i}\right):=f:=\sum_{i=1}^{m} U\left(y_{i}\right) u_{i} .
$$

Dann ist $V \pi$-isometrisch:

$$
\begin{aligned}
{\left[V f^{\prime} \mid V f^{\prime}\right] } & =\sum_{i, j=1}^{m}\left[U\left(y_{i}\right)\left(K(\cdot, e) u_{i}\right) \mid U\left(y_{j}\right)\left(K(\cdot, e) u_{j}\right)\right] \\
& =\sum_{i, j=1}^{m}\left[K\left(y_{j}, y_{i}\right) u_{i} \mid u_{j}\right]=[f \mid f] .
\end{aligned}
$$

Mann sieht leicht, daß $V: \mathfrak{\Omega}^{\prime} \rightarrow \mathfrak{Q}$ bijektiv ist. Demzufolge läßt $V$ sich stetig und $\pi$-isometrisch auf $\tilde{E}^{\prime}$ fortsetzen, denn $\mathfrak{\Omega}^{\prime}$ (bzw. $\mathfrak{\Omega}$ ) liegt dicht in $\mathfrak{E}^{\prime}$ (bzw. $\left.\mathfrak{E}\right)$. Für $f^{\prime} \in \mathfrak{Q}^{\prime}$ gilt weiter

$$
\begin{aligned}
V^{-1} U(y) V f^{\prime} & =V^{-1} U(y) f=V^{-1}\left(\sum_{i=1}^{m} U\left(y y_{i}\right) u_{i}\right) \\
& =\sum_{i=1}^{m} U^{\prime}\left(y y_{i}\right) u_{i}=U^{\prime}(y) f^{\prime},
\end{aligned}
$$

woraus die Behauptung folgt.

4.3. Darstellungen von Gruppen. Wie in 4.2 sei (S) ein $\pi_{x}$-Raum. Weiter setzen wir voraus, daß $\mathfrak{X}$ eine Gruppe ist. Das folgende Resultat ist eine Verallgemeinerung von Theorem 1 aus [12], S. 401.

Satz 4.3. Jeder $\pi$-unitären Darstellung $U$ der Gruppe $\mathfrak{X}$ in (S) entspricht ein $\mathfrak{G}-K$ ern $K$ auf $\mathfrak{X}$ mit den Eigenschaften

1) $K$ hat genau $\approx$ negative Quadrate;

2) $K$ ist linksinvariant, d.h. für alle $x, y, z \in \mathfrak{X}$ gilt

$$
K(z x, z y)=\bar{K}(x, y)
$$

und

3) $K(e, e)=I$.

Umgekehrt entspricht jedem $\mathfrak{3}-K e r n \quad K$ auf $\mathfrak{X}$ mit den Eigenschaften 1)-3) eine $\pi$-unitäre Darstellung $U$ von $\mathfrak{X}$ in einem $\mathfrak{G S}$ umfassenden $\pi_{*}$-Raum E.

Diese Zuordnung ist durch 


$$
K(x, y)=U\left(x^{-1} y\right)
$$

für alle $x, y \in \mathfrak{X}$ gegeben.

Beweis. A. Wenn $\mathfrak{X}$ eine $\pi$-unitäre Darstellung $U$ in $\mathbb{S}$ hat, so

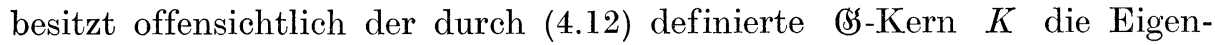
schaften 1)-3).

B. Es sei nun ein (SS-Kern $K$ mit den Eigenschaften 1)-3) gegeben. Nach Satz 3.3 existiert dann ein $\pi_{\varkappa}$-Raum $\mathfrak{E} \subset \mathcal{F}(\mathfrak{X} ;(\mathfrak{S})$ mit reproduzierendem Kern $K$. Wie im Beweis von Satz 4.2 betten wir (5s in \& durch $u \mapsto K(\cdot, e) u$ ein und definieren auf dem Teilraum $\mathfrak{\&}$ aller Abbildungen $f: \mathfrak{X} \rightarrow \mathscr{G S}$ der Form

$$
f=\sum_{i=1}^{m} K\left(\cdot, y_{i}\right) u_{i}
$$

einen Operator $U(y)$ durch (4.7). Für $U(y)$ gelten dann die Beziehungen $(4.8)-(4.10)$.

Wir zeigen noch, daß $U(y) \pi$-isometrisch ist. Aus (4.11) folgt für $f, g \in \mathfrak{Q}$

$$
\begin{aligned}
& {[U(y) f \mid U(y) g]=\left[\sum_{i=1}^{m} K\left(\cdot, y y_{i}\right) u_{i} \mid \sum_{j=1}^{n} K\left(\cdot, y z_{j}\right) u_{j}\right]} \\
& =\sum_{i=1}^{m} \sum_{j=1}^{n}\left[K\left(y z_{j}, y y_{i}\right) u_{i} \mid v_{j}\right]=\sum_{i=1}^{m} \sum_{j=1}^{n}\left[K\left(z_{j}, y_{i}\right) u_{i} \mid v_{j}\right]=[f \mid g] .
\end{aligned}
$$

Wir können also $U(y)$ zu einem $\pi$-unitären Operator in $\mathcal{B}(\mathfrak{E})$ fortsetzen, denn $\mathfrak{\Omega}$ liegt dicht in $\mathfrak{E}$. Demzufolge ist $U: \mathfrak{X} \rightarrow \Omega(\mathfrak{S})$ eine $\pi$-unitäre Darstellung.

Aus (4.11) folgt für alle $x, y \in \mathfrak{X}$ und $u, v \in \mathbb{B S}$

$$
\begin{aligned}
& {\left[U\left(x^{-1} y\right) u \mid v\right]=\left[K\left(\cdot, x^{-1} y\right) u \mid K(\cdot, e) v\right]} \\
& =\left[K\left(x^{-1} x, x^{-1} y\right) u \mid v\right]=[K(x, y) u \mid v],
\end{aligned}
$$

also gilt (4.12).

Folgerung 4.4. Es sei $T: \mathfrak{X} \rightarrow \mathscr{B}($ SS) eine Abbildung mit $T(e)=I$ und $T\left(x^{-1}\right)=T(x)^{+}$für alle $x \in \mathfrak{X}$.

Hat der durch

$$
K(x, y):=T\left(x^{-1} y\right)
$$

für alle $x, y \in \mathfrak{X}$ definierte $\mathbb{S}$-Kern $K$ auf $\mathfrak{X}$ genau * negative Quadrate, so existieren ein (S) umfassender $\pi_{\varkappa}$-Raum [5 mit dem reproduzierenden Kern $K$ und eine $\pi$-unitäre Darstellung $U$ von $\mathfrak{X}$ in $\mathfrak{F}$ mit

$$
T(x)=\left.P_{\mathfrak{G}} U(x)\right|_{\mathfrak{G}} \quad(x \in X)
$$

und

$$
\mathfrak{E}=\text { a. 1. H. }\{U(x) u \mid x \in \mathfrak{X}, u \in \mathbb{F}\} .
$$


Diese Darstellung ist bis auf eine Isomorphie eindeutig bestimmt.

In der Tat, der (3)-Kern $K$ erfüllt offensichtlich die Bedingungen 1)-3) von Satz 4.3 und demzufolge auch die Voraussetzungen von Satz 4.2.

Im Falle eines Hilbertraumes ist diese Folgerung in [17] (Theorem 7.1, S. 25) dargestellt.

Aus Folgerung 4.4 erhalten wir die folgende Erweiterung eines Satzes von B. Sz.-Nagy [16] (siehe auch [17], S. 28-29): ${ }^{4}$ )

Satz 4.5. Ist $V \in B_{3}\left(\Pi_{x}\right)$ ein $\pi$-kontrahierender Operator im Pontrjaginraum $\Pi_{\varkappa}$, so besitzt $V$ eine minimale $\pi$-unitäre Dilatation; d.h. es existieren ein $\pi_{*}$-Raum $\tilde{\Pi}_{\varkappa} \supset \Pi_{*}$ und ein $\pi$-unitärer Operator $U \in \mathscr{C}_{(}\left(\tilde{\Pi}_{*}\right)$ so, daß gilt:

$$
\begin{gathered}
V^{n}=\left.\tilde{P} U^{n}\right|_{I_{\varkappa}} \quad(n=0,1,2, \ldots) ; \\
\tilde{I}_{\varkappa}=\text { a.l. H. }\left\{U^{n} u \mid u \in \Pi_{\varkappa}, n=0, \pm 1, \ldots\right\} .
\end{gathered}
$$

Dabei ist $\tilde{P}$ der $\pi$-orthogonale Projektor von $\tilde{\Pi}_{\varkappa}$ auf $\Pi_{\varkappa}$.

In Folgerung 4.4 wird für $\mathfrak{X}$ die (additive) Gruppe $Z$ aller ganzen Zahlen und für $T$ die durch

$$
T(n):= \begin{cases}V^{n} & \text { für } n \geq 1, \\ 0 & \text { für } n=0, \\ \left(V^{+}\right)^{-n} & \text { für } n \leq 1\end{cases}
$$

definierte Abbildung $T: \mathfrak{X} \rightarrow \mathscr{S}_{\left(\Pi_{\varkappa}\right)}$ gewählt. Dann kann man wie in [17], S. 28-29, zeigen, daß für den zu $T$ gehörenden Kern $K$ gilt

$$
\sum_{i, j=1}^{n}\left[K\left(n_{i}, n_{j}\right) u_{j} \mid u_{i}\right]=\left[v_{0} \mid v_{0}\right]+\sum_{m=1}^{\infty}\left[\left(I-V^{+} V\right) v_{m} \mid v_{m}\right] ;
$$

dabei ist $\left(v_{m}\right)$ eine beliebige Folge in $\Pi_{\varkappa}$ derart, daß $v_{m}=0$ nur für endlich viele Indizes. Da $V \pi$-kontrahierend ist, folgt daraus, daß $K$ genau $\varkappa$ negative Quadrate hat. Aus Folgerung 4.4 folgt nun die Behauptung, wenn man nur $U:=U(1)$ setzt.

\section{Verallgemeinerte Resolventen eines $\pi$-hermiteschen Operators}

5.1. Grundbegriffe. Als zweite Anwendung der Theorie betrachten wir jetzt verschiedene verallgemeinerte Resolventen eines $\pi$-hermiteschen Operators. Zuerst seien einige Definitionen und Resultate gegeben. (Siehe insbesondere [7].)

$\left.{ }^{4}\right)$ Herr Heinz Langer hat dem Verfasser freundlicherweise mitgeteilt, daß D. Z. Arov einen anderen (unveröffentlichten) Beweis für dieses Resultat gegeben hat. 
In diesem Kapitel ist $\Pi_{\varkappa}$ immer ein $\pi_{\varkappa}$-Raum und $A$ ein in $\Pi_{\varkappa}$ dicht definierter abgeschlossener $\pi$-hermitescher Operator.

Der Operator $A$ besitzt $\pi$-selbstadjungierte Erweiterungen, die möglicherweise in einem Oberraum $\tilde{\Pi}_{\varkappa}$ von $\Pi_{\varkappa}$ wirken. Das Spektrum jeder $\pi$-selbstadjungierten Erweiterung $\tilde{A}$ von $A$ liegt im Streifen

$$
\Sigma_{A}:=\left\{z \in C|| \operatorname{Im} z \mid \leq h_{A}\right\},
$$

wobei $h_{A}>0$ nur von $A$ abhängt. Außerhalb von $\Sigma_{A}$ gilt die Absetzung

$$
\left\|(\tilde{A}-z I)^{-1}\right\| \leq 1 /\left(|\operatorname{Im} z|-h_{A}\right) .
$$

Es sei $\tilde{\Pi}_{\varkappa}$ ein Oberraum von $\Pi_{\varkappa}$ und $\tilde{A}$ ein $\pi$-selbstadjungierter Operator in $\tilde{\Pi}_{\varkappa}$. Ist $\tilde{R}$ die Resolvente von $\tilde{A}$, d.h. $\tilde{R}(z):=(\tilde{A}-z \tilde{I})^{-1}$ $\in \mathcal{G}\left(\tilde{\Pi}_{\varkappa}\right)$ für $z \in \varrho(\tilde{A})$, und $\tilde{P}$ der $\pi$-orthogonale Projektor von $\tilde{\Pi}_{x}$ auf $\Pi_{r}$, so heißt die durch

$$
R(z):=\left.\tilde{P} \tilde{R}(z)\right|_{I_{\gamma}} \quad(z \in \varrho(\tilde{A}))
$$

definierte Funktion $R$ eine verallgemeinerte Resolvente des $\pi$-selbstadjungierten Operators $\tilde{A}$. Wenn $\tilde{A}$ speziell eine Erweiterung von $A$ ist, so nennen wir $R$ eine verallgemeinerte Resolvente des $\pi$-hermiteschen Operators $A$.

Wir wählen ein $\alpha>h_{A}$ und setzen $\Pi_{r}^{+}:=\mathfrak{D}\left(A^{+}\right)$, versehen mit dem $\pi$-Skalarprodukt

$$
[u \mid v]_{+}:=\alpha^{2}[u \mid v]+\left[A^{+} u \mid A^{+} v\right] \quad\left(u, v \in \mathfrak{D}\left(A^{+}\right)\right) .
$$

Dann ist nach [14] $\Pi_{\varkappa}^{+}$ein Pontrjaginraum von Grundelementen bezüglich $\Pi_{\varkappa}$. Sei $\Pi_{\varkappa}^{-}$der zugehörige Pontrjaginraum verallgemeinerter Elemente.

Ist $R$ eine verallgemeinerte Resolvente von $A$, so liegt $R(z)$ in $B\left(\Pi_{\varkappa} ; \Pi_{\varkappa}^{+}\right)$; demzufolge können wir die zugehörige erweiterte verallgemeinerte Resolvente $\hat{R}$ durch die Gleichung

$$
\hat{R}(z):=R(\bar{z})^{\oplus} \quad(z \in \varrho(\tilde{A}))
$$

definieren, d.h. es gilt $\hat{R}(z) \in \widehat{\partial}\left(\Pi_{\varkappa}^{-} ; \Pi_{\varkappa}\right)$ und

$$
[\hat{R}(z) x \mid f]=[x \mid R(\bar{z}) f]
$$

für alle $x \in \Pi_{x}^{-}, f \in \Pi_{x}$. Dann ist $R(z)$ die Erweiterung des zunächst auf $\Pi_{\varkappa}$ definierten Operators $R(z)$ auf $\Pi_{\varkappa}^{-}$durch Stetigkeit.

Wir wollen nun die oben eingeführten verschiedenen verallgemeinerten Resolventen charakterisieren. 


\subsection{Verallgemeinerte Resolventen eines $\pi$-selbstadjungierten Operators.} Im Folgenden sei $M>0$ eine Konstante und

$$
\mathfrak{U}(i \infty):=\{i t \mid t>M\} .
$$

Der folgende Satz verallgemeinert ein entsprechendes Resultat von R. Shonkwiler [13]; man vergleiche auch [9] und [18].

Satz 5.1. Ist $R$ eine verallgemeinerte Resolvente eines $\pi$-selbstadjungierten Operators $\tilde{A}$ in $\tilde{\Pi}_{\varkappa} \supset \Pi_{\varkappa}$, so gilt:

1) Der durch

$$
K(z, \zeta):=\frac{\bar{z} \zeta}{\bar{z}-\zeta}\left(R(z)^{+}-R(\zeta)\right)
$$

definierte $\Pi_{\varkappa}-K e r n \quad K$ auf $\mathfrak{H}(i \infty)$ (mit $M:=2 h_{\tilde{A}}$ ) hat $\varkappa^{\prime}$ negative Quadrate für ein gewisses $x^{\prime}$ mit $0 \leq \varkappa^{\prime} \leq \varkappa$.

2) Für jedes $f \in \Pi_{\varkappa}$ konvergiert $(-i t) R(i t) f$ schwach gegen $f$, wenn $t \rightarrow \infty$.

Umgekehrt, hat eine Funktion $R: \mathfrak{U}(i \infty) \rightarrow \mathcal{B}\left(I_{x}\right)$ die Eigenschaften 1) -2), so existiert eine verallgemeinerte Resolvente eines $\pi$-selbstadjungierten Operators, die mit $R$ in $\mathfrak{H}(i \infty)$ zusammenfällt.

Beweis. A. Sei zuerst $R$ eine verallgemeinerte Resolvente eines $\pi$-selbstadjungierten Operators $\tilde{A}$, d.h. (5.2) gilt.

Um die Eigenschaft 1) zu zeigen bemerken wir zuerst, daß aus der Hilbertschen Relation unmittelbar die Beziehung:

$$
R(\bar{z})-R(\zeta)=\left.(\bar{z}-\zeta) \tilde{P} \tilde{R}(\bar{z}) \tilde{R}(\zeta)\right|_{I I_{\varkappa}}
$$

folgt. Dies ergibt

$$
\left[K\left(z_{i}, z_{j}\right) f_{j} \mid f_{i}\right]=\bar{z}_{i} z_{j}\left[\tilde{P} \tilde{R}\left(\bar{z}_{i}\right) \tilde{R}\left(z_{j}\right) f_{j} \mid f_{i}\right]=\left[z_{j} \tilde{R}\left(z_{j}\right) f_{j} \mid z_{i} \tilde{R}\left(z_{i}\right) f_{i}\right]
$$

für alle $\left(z_{i}\right)_{1 \leqq i \leqq n}$ in $\mathfrak{U}(i \infty)$ und $\left(f_{i}\right)_{1 \leqq i \leqq n}$ in $\Pi_{\%}$. (Dabei setzen wir $M:=2 h_{\tilde{A}}$.) Somit hat der $\Pi_{\varkappa}$-Kern $K$ höchstens * negative Quadrate.

Seien nun $\tilde{f} \in \tilde{I}_{\varkappa}$ und $\tilde{g} \in \mathfrak{D}(\tilde{A})$ beliebig. Aus (1.3) und (5.1) erhält man

$$
\begin{aligned}
|[((-i t) \tilde{R}(i t)-I) \tilde{f} \mid \tilde{g}]| & =|[-\tilde{A} \tilde{R}(i t) \tilde{f} \mid \tilde{g}]| \leq\|\tilde{R}(i t)\|\|\tilde{f}\|\|\tilde{A} \tilde{g}\| \\
& \leq\left(t-h_{\tilde{A}}\right)^{-1}\|\tilde{f}\|\|\tilde{A} \tilde{g}\| \rightarrow 0,
\end{aligned}
$$

wenn $t \rightarrow \infty$. Für jedes $\tilde{f} \in \tilde{\Pi}_{\varkappa}$ ist die Menge $\{-i t \tilde{R}(i t) \tilde{f} \mid t>M\}$ auch beschränkt:

$$
\|-i t \tilde{R}(i t) \tilde{f}\| \leq t\left(t-h_{\tilde{A}}\right)^{-1}\|\tilde{f}\|<2\|\tilde{f}\|
$$

Da $\mathscr{D}(\tilde{A})$ dicht in $\tilde{\Pi}_{\varkappa}$ liegt, folgt $-i t \tilde{R}(i t) \tilde{f} \rightarrow \tilde{f}$ und auf Grund von (5.2) auch $-i t R(i t) f \rightarrow f$ für alle $f \in \Pi_{x}$. 
B. Wir beweisen jetzt die Umkehrung. Dazu konstruieren wir einen Pontrjaginraum $\tilde{\Pi}_{\varkappa} \supset \Pi_{\varkappa}$ und einen $\pi$-selbstadjungierten Operator $\tilde{A}$ in $\tilde{\Pi}_{\varkappa}$ derart, daß (5.2) gilt in $\mathfrak{H}(i \infty)$.

Nach Satz 3.3 existiert ein $\pi_{\varkappa^{\prime}}$ Raum $\Pi_{\varkappa^{\prime}}^{0} \subset \mathcal{H}\left(\mathfrak{U}(i \infty) ; \Pi_{\varkappa}\right)$ mit reproduzierendem Kern $K$. Der Teilraum $\mathfrak{L}$ von $\Pi_{\varkappa^{\prime}}^{0}$ habe dieselbe Bedeutung wie in Satz 3.3.

Wir zeigen, daß für jedes $f \in \Pi_{r}$ die Folge $(K(\cdot, i n) f), n>M$, in $\mathbb{Q}$ eine Cauchyfolge ist. In der Tat, für jedes $K(\cdot, \zeta) g$ in $\mathbb{\Omega}$ gilt nach Voraussetzungen

$$
\begin{aligned}
& {[K(\cdot, i n) f-K(\cdot, i m) f \mid K(\cdot, \zeta) g]} \\
& =\left[\left\{\frac{\bar{\zeta} i n}{\bar{\zeta}-i n}\left(R(\zeta)^{+}-R(i n)\right)-\frac{\bar{\zeta} i m}{\bar{\zeta}-i m}\left(R(\zeta)^{+}-R(i m)\right)\right\} f \mid g\right] \\
& =\bar{\zeta}\left\{\left(\frac{\bar{\zeta}}{i n}-1\right)^{-1}-\left(\frac{\bar{\zeta}}{i m}-1\right)^{-1}\right\}[f \mid R(\zeta) g] \\
& \quad+\bar{\zeta}(\bar{\zeta}-i n)^{-1}[(-i n) R(i n) f \mid g] \\
& \quad-\bar{\zeta}(\bar{\zeta}-i m)^{-1}[(-i m) R(i m) f \mid g] \rightarrow 0,
\end{aligned}
$$

wenn $m, n \rightarrow \infty$. Analog erhält man

$$
[K(i n, i n) f \mid f]=\frac{1}{2}\left[\left(i n R(i n)^{+}+(-i n) R(i n)\right) f \mid g\right] \rightarrow 0
$$

und

$$
\begin{aligned}
& {[K(i m, i n) f \mid f]} \\
& =(m+n)^{-1}\left\{n\left[i m R(i m)^{+} f \mid f\right]+m[(-i n) R(i n) f \mid f]\right\} \rightarrow 0,
\end{aligned}
$$

wenn $m, n \rightarrow \infty$. Daraus folgt für $m, n \rightarrow \infty$

$$
\begin{aligned}
& {[(K(\cdot, i n)-K(\cdot, i m)) f \mid(K(\cdot, i n)-K(\cdot, i m)) f]} \\
& =[K(i n, i n) f \mid f]+[K(i m, i m) f \mid f]-2 \operatorname{Re}[K(i m, i n) f \mid f] \rightarrow 0 .
\end{aligned}
$$

Nach dieser Beziehung und (5.6) ist $(K(\cdot, i n) f)$ eine Cauchyfolge, die in $\Pi_{\varkappa^{\prime}}^{0}$ konverigiert. Der Grenzwert wird mit $K(\cdot, i \infty) f$ bezeichnet.

Für $K(\cdot, i \infty) f \in \Pi_{\varkappa^{\prime}}^{0}$ und $K(\cdot, z) g \in \Omega$ gilt

$$
[K(\cdot, z) g \mid K(\cdot, i \infty) f]=\lim _{n \rightarrow \infty}[K(i n, z) g \mid f]=-z[R(z) g \mid f]
$$

und

$$
\begin{aligned}
{[K(\cdot, i \infty) g \mid K(\cdot, i \infty) f] } & =\lim _{n \rightarrow \infty}[K(\cdot, i n) g \mid K(\cdot, i \infty) f] \\
& =\lim _{n \rightarrow \infty}(-i n)[R(i n) g \mid f]=[g \mid f] .
\end{aligned}
$$


Deshalb können wir den Raum $\Pi_{\varkappa}$ in $\Pi_{\varkappa^{\prime}}^{0}$ einbetten durch die Identifizierung $f \mapsto K(\cdot, i \infty) f$. Dann ist notwendig $x \leq x^{\prime}$ und somit weil in 1) $x^{\prime} \leq x$ vorausgesetzt wurde, schließlich $x^{\prime}=\varkappa$.

Man kann weiter wie in [13] einen $\pi$-hermiteschen Operator in $I I_{*}^{0}$ bilden: Für $\varphi:=\sum_{j=1}^{n} K\left(\cdot, z_{j}\right) f_{j} \in \mathfrak{Q}$ setzt man

$$
A \varphi:=\sum_{j=1}^{n} z_{j}\left\{K\left(\cdot, z_{j}\right)-K(\cdot, i \infty)\right\}, f_{j} .
$$

Es läßt sich leicht zeigen, daß $A$ ein in $\Pi_{*}^{0}$ dicht definierter $\pi$ hermitescher Operator ist, den wir o. B.d.A. als abgeschlossen voraussetzen können.

Wir wollen jetzt (5.2) zeigen. Dazu bilden wir für $z \in \mathfrak{H}(i \infty)$ den Operator $A-z I$ :

$$
(A-z I)(K(\cdot, \zeta) f)=(z-\zeta) K(\cdot, \zeta) f-\zeta K(\cdot, i \infty) f
$$

für $K(\cdot, \zeta) f \in \mathfrak{L}$. Insbesondere gilt

$$
(A-z I)(K(\cdot, z) f)=-z K(\cdot, i \infty) f .
$$

Wählt $\operatorname{man} M>0$ so groß, daß $M>h_{A}$ ist, so erhält man daher

$$
(A-z I)^{-1}(K(\cdot, i \infty) f)=-z^{-1} K(\cdot, z) f .
$$

Wir erweitern $A$ zu einem $\pi$-selbstadjungierten Operator $\tilde{A}$, der in einem Oberraum $\tilde{\Pi}_{\varkappa}$ von $\Pi_{\kappa}^{0}$ wirkt. Dann folgt aus (5.7) und (5.8)

$$
\begin{aligned}
{[R(z) f \mid g] } & =-z^{-1}[-z R(z) f \mid g]=-z^{-1}[K(\cdot, z) f \mid K(\cdot, i \infty) g] \\
& =\left[(A-z I)^{-1}(K(\cdot, i \infty) f) \mid K(\cdot, i \infty) g\right] \\
& =\left[\tilde{P}(\tilde{A}-z \tilde{I})^{-1}(K(\cdot, i \infty) f) \mid K(\cdot, i \infty) g\right],
\end{aligned}
$$

wobei $\tilde{P}$ den $\pi$-orthogonalen Projektor von $\tilde{\Pi}_{\varkappa}$ auf $\Pi_{\varkappa}$ bezeichnet. Dies bedeutet, daß $R$ mit der verallgemeinerten Resolvente $\left.\tilde{P}(\tilde{A}-z \tilde{I})^{-1}\right|_{\Pi_{r}}$ von $A$ in $\mathfrak{H}(i \infty)$ übereinstimmt.

5.3. Verallgemeinerte Resolventen eines $\pi$-hermiteschen Operators. $\mathrm{Al}$ Spezialfall erhalten wir aus Satz 5.1

Satz 5.2. Ist $R$ eine verallgemeinerte Resolvente eines $\pi$-hermiteschen abgeschlossenen Operators $A$ in $\Pi_{\%}$, so hat sie Eigenschaften 1) und 2) aus Satz 5.1 (mit $M:=2 h_{A}$ ) und

3) es existieren ein Punkt $w \in \mathfrak{H}(i \infty)$ und ein Teilraum $\mathfrak{M} \subset \Pi_{\varkappa}$ derart, daß $\mathfrak{R}(w)$ auf $\mathfrak{M}$ injektiv ist, $\mathfrak{R}(w) \mathfrak{M}$ dicht in $\Pi_{*}$ liegt und Beziehung 


$$
R(w)^{+} f-R(w) f=(\bar{w}-w) R(w)^{+} R(w) f
$$

für alle $f \in \mathfrak{M}$ besteht.

Umgekehrt, hat eine Funktion $R: \mathfrak{U}(i \infty) \rightarrow \mathscr{B}\left(\Pi_{*}\right)$ die Eigenschaften 1)-3), so existiert eine verallgemeinerte Resolvente eines $\pi$-hermiteschen abgeschlossenen Operators in $\Pi_{*}$, die mit $R$ in $\mathfrak{U}(i \infty)$ zusammenfällt.

Beweis. A. Ist $R$ eine verallgemeinerte Resolvente eines $\pi$-hermiteschen abgeschlossenen Operators, so ist $R$ insbesondere eine verallgemeinerte Resolvente einer $\pi$-selbstadjungierten Erweiterung $\tilde{A}$ von $A$. Also hat $R$ nach Satz 5.1 Eigenschaften 1) und 2).

Um 3) zu beweisen wählen wir ein beliebiges $w$ aus $\mathfrak{H}(i \infty)$. Setzt man $\mathfrak{M}:=\mathfrak{R}(A-w I)$, so sieht man leicht, daß $\left.\Re(w)\right|_{\mathfrak{M}}=(A-w I)^{-\mathbf{1}}$ gilt. Aus dieser Relation folgt unmittelbar Eigenschaft 3$)$.

B. Wir zeigen nun die Umkehrung. Dazu setzen wir $\mathfrak{D}(A):=\mathfrak{R}(w) \mathfrak{M}$ und

$$
A f:=R(w)^{-1} f+w f \quad(f \in \mathfrak{I}(A)) .
$$

Dann ist $A$ ein dicht definierter, abgeschlossener Operator in $\Pi_{\%}$.

Der Operator $A$ ist sogar $\pi$-hermitesch. In der Tat, für alle Elemente $f=R(w) f_{1}$ und $g=R(w) g_{1}$ von $\mathfrak{D}(A)$ mit $f_{1}, g_{1} \in \mathfrak{M}$ gilt nach (5.10) und $(5.9)$

$$
\begin{aligned}
{[A f \mid g] } & =\left[f_{1} \mid g\right]+w[f \mid g] \\
& =\left[\left(R(w)^{+}-R(w)+R(w)\right) f_{1} \mid g_{1}\right]+w[f \mid g] \\
& =(\bar{w}-w)\left[R(w) f_{1} \mid R(w) g_{1}\right]+\left[f \mid g_{1}\right]+w[f \mid g] \\
& =\bar{w}[f \mid g]+\left[f \mid g_{1}\right]=[f \mid A g] .
\end{aligned}
$$

Andererseits wissen wir aus Satz 5.1, daß $R$ eine verallgemeinerte Resolvente eines $\pi$-selbstadjungierten Operators $\tilde{A}$ in $\tilde{\Pi}_{\%}$ ist, d. h. (5.2) gilt. Es bleibt deshalb nur noch zu zeigen, daß $\tilde{A}$ eine Erweiterung von $A$ ist.

$\mathrm{Zu}$ diesem $\mathrm{Zweck}$ beweisen wir

$$
\left.\tilde{R}(w)\right|_{\mathfrak{m}}:=\left.(\tilde{A}-w \tilde{I})^{-1}\right|_{\mathfrak{M}}=\left.R(w)\right|_{\mathfrak{M}} \cdot
$$

Sei also $f_{1}=R(w)^{-1} f \in \mathfrak{M} \quad$ mit $f \in \mathfrak{D}(A)$ beliebig und $\quad \tilde{f}_{1}:=\tilde{R}(w) f_{1}$. Da $A \pi$-hermitesch ist, erhalten wir

$$
\begin{aligned}
0 & =\operatorname{Im}[A f \mid f]=\operatorname{Im}\left\{\left[f_{1} \mid R(w) f_{1}\right]+w[f \mid f]\right\} \\
& =\operatorname{Im}\left\{\left[(\tilde{A}-w \widetilde{I}) \tilde{f}_{1} \mid \tilde{f}_{1}\right]+w[f \mid f]\right\}=(\operatorname{Im} w)\left\{[f \mid f]-\left[\tilde{f}_{1} \mid \tilde{f}_{1}\right]\right\} .
\end{aligned}
$$

Somit gilt für alle $f_{1} \in \mathfrak{M}$

$$
\left[R(w) f_{1} \mid R(w) f_{1}\right]=\left[\tilde{R}(w) f_{1} \mid \tilde{R}(w) f_{1}\right] .
$$


Schreiben wir $\tilde{R}(w)$ in der Form

$$
\tilde{R}(w) f_{1}=(\tilde{I}-\tilde{P}) \tilde{R}(w) f_{1}+R(w) f_{1}
$$

für $f_{1} \in \mathfrak{M}$, so folgt aus (5.12)

$$
\left[(\tilde{I}-\tilde{P}) \tilde{R}(w) f_{1} \mid(\tilde{I}-\tilde{P}) \tilde{R}(w) f_{1}\right]=0
$$

für alle $f_{1} \in \mathfrak{M}$. Auf dem Teilraum $\Pi_{\varkappa}^{\perp}:=\left\{\tilde{f} \in \tilde{\Pi}_{\varkappa} \mid \tilde{f} \perp \Pi_{\varkappa}\right\}$ ist aber das $\pi$-Skalarprodukt $[\cdot \mid \cdot]$ positiv definit und daher gilt

$$
\left.(\tilde{I}-\tilde{P}) \tilde{R}(w)\right|_{\mathfrak{m}}=O,
$$

woraus (5.11) folgt.

Aus (5.11) und (5.10) ergibt sich unmittelbar, daß $\tilde{A}$ eine $\pi$-selbstadjungierte Erweiterung von $A$ ist. Somit ist Satz 5.2 bewiesen.

5.4. Verallgemeinerte Resolventen für vorgegebenen Operator. Wenn wir noch spezieller fragen, wann eine Funktion $R$ eine verallgemeinerte Resolvente eines vorgegebenen abgeschlossenen $\pi$-hermiteschen Operators $A$ ist, liefert Satz 5.2 die folgende Antwort:

Satz 5.3. Sei $A$ ein abgeschlossener $\pi$-hermitescher Operator in $\Pi_{\%}$. Dann hat jede verallgemeinerte Resolvente $R$ von $A$ die Eigenschaften 1)-2) aus Satz 5.1 und

$\left.3^{\prime}\right) \quad R(w)(A-w I) f=R(w)^{+}(A-\bar{w} I) f=f \quad$ für ein $\quad w \in \mathfrak{U}(i \infty)$ und alle $f \in \mathfrak{D}(A)$.

Umgekehrt, hat eine Funktion $R: \mathfrak{U}(i \infty) \rightarrow B_{(}\left(\Pi_{r}\right)$ diese Eigenschaften, so existiert eine verallgemeinerte Resolvente von $A$, die mit $R$ in $\mathfrak{H}(i \infty)$ zusammenfällt.

Beweis. A. Die Notwendigkeit aller Bedingungen folgt unmittelbar aus der Relation $R(w) f=(A-w I)^{-1} f, \quad f \in \Re(A-w I)$, und aus Satz 5.2.

B. Wir zeigen daher die Hinlänglichkeit der Bedingungen 1)-2) und $\left.3^{\prime}\right)$. Zuerst wählen wir die Konstante $M>h_{A}$. Sei $w \in \mathfrak{H}(i \infty)$ fixiert und $\mathfrak{M}:=\mathfrak{R}(A-w I)$. Dann folgt aus $\left.3^{\prime}\right)$ für alle $f \in \mathfrak{M}$

$$
R(w) f=R(w)(A-w I)(A-w I)^{-1} f=(A-w I)^{-1} f .
$$

Der Operator $\left.R(w)\right|_{\mathscr{M}}$ ist also injektiv und $R(w) \mathfrak{M}=\mathfrak{D}(A)$ liegt dicht in $\Pi_{\varkappa}$. Auf Grund von (5.13) und $3^{\prime}$ ) gilt weiter

$$
\begin{aligned}
R(w)^{+} f & =R(w)^{+}(A-w I) R(w) f, \\
R(w) f & =R(w)^{+}(A-\bar{w} I) R(w) f
\end{aligned}
$$

für alle $f \in \mathfrak{M}$. Substrahieren wir diese Gleichungen voneinander, so ergibt sich Relation (5.9). 
Nach Satz 5.2 ist $R$ also eine verallgemeinerte Resolvente eines $\pi$ hermiteschen Operators $A_{1}$ in $\Pi_{\varkappa}$. Dieser Operator ist durch

$$
A_{1} f:=R(w)^{-1} f+w f, f \in \mathfrak{D}\left(A_{1}\right):=R(w) \mathfrak{M},
$$

gegeben. Weiter stimmt $A_{1}$ mit $A$ überein. In der Tat, $\mathscr{D}\left(A_{1}\right)=R(w) \mathfrak{M}$ $=\mathfrak{D}(A)$ und nach (5.13) gilt

$$
A_{1} f=R(w)^{-1} f+w f=(A-w I) f+w f=A f
$$

für alle $f \in \mathfrak{D}\left(A_{1}\right)=\mathfrak{D}(A)$. Satz 5.3 ist damit bewiesen.

Die Sätze 5.2 und 5.3 verallgemeinern einige Resultate von A. V. Štraus [15]; er betrachtet aber auch nicht dicht definierte Operatoren.

5.5. Erweiterte verallgemeinerte Resolventen. Wir charakterisieren noch die erweiterten varallgemeinerten Resolventen eines $\pi$-hermiteschen Operators.

Satz 5.4. Seien $A$ ein abgeschlossener $\pi$-hermitescher Operator im Pontrjaginraum $\Pi_{\%}, \Pi_{*}^{+}$der aus $\mathfrak{D}\left(A^{+}\right)$gebildete Pontrjaginraum von Grundelementen und $\Pi_{*}^{-}$der zugehörige Pontrjaginraum verallgemeinerter Elemente.

Jede erweiterte verallgemeinerte Resolvente $\hat{R}$ von $A$ hat die folgenden Eigenschaften:

1) Die Abbildung $\left.\lambda \mapsto \hat{R}(\lambda)\right|_{I_{\varkappa}}$ ist eine verallgemeinerte Resolvente eines abgeschlossenen $\pi$-hermiteschen Operators in $\Pi_{*}$.

2) Es gibt ein $w \in \mathfrak{H}(i \infty)$ derart, daß

$$
\hat{R}(w)(A-w I) u=u
$$

für alle $u \in \mathfrak{D}(A)$ gilt.

Umgekehrt, hat eine Abbildung $\hat{R}: \mathfrak{H}(i \infty) \rightarrow \mathrm{B}\left(\Pi_{r}^{-} ; \Pi_{,}\right)$die Eigenschaften 1)-2), so existiert eine erweiterte verallgemeinerte Resolvente von $A$, die mit $\hat{R}$ in $\mathfrak{U}(i \infty)$ übereinstimmt.

Beweis. A. Die Notwendigkeit ist klar, denn $\left.\lambda \mapsto \hat{R}(\lambda)\right|_{I I_{\varkappa}}$ ist eine verallgemeinerte Resolvente des Operators $A$.

B. Umgekehrt, sei $\left.\lambda \mapsto \hat{R}(\lambda)\right|_{I_{\varkappa}}$ eine verallgemeinerte Resolvente eines abgeschlossenen $\pi$-hermiteschen Operators $A_{1}$ in $\Pi_{\varkappa}$ derart, daß (5.14) gilt. Dann existiert eine $\pi$-selbstadjungierte Erweiterung $\tilde{A}_{1}$ von $A$ in $\tilde{\Pi}_{\varkappa}$ mit der Eigenschaft

$$
\hat{R}(\lambda) f=\tilde{P}\left(\tilde{A}_{1}-\lambda \tilde{I}\right)^{-1} f
$$

für alle $f \in \Pi_{r}$. Diese Gleichung besteht zuerst nur in $\mathfrak{U}(i \infty)$, aber wir können $\left.\lambda \mapsto \hat{R}(\lambda)\right|_{I_{\varkappa}}$ durch diese Relation für alle $\lambda \in \varrho\left(\widetilde{A}_{1}\right)$ definieren.

Aus (5.14) und (5.15) folgt für alle $u \in \mathfrak{D}(A)$ 


$$
\begin{aligned}
u & =\hat{R}(w)(A-w I) u \\
& =\mathcal{P}\left(\tilde{A}_{1}-w \tilde{I}\right)^{-1}\left(A_{1}-w I\right)\left(A_{1}-w I\right)^{-1}(A-w I) u \\
& =\left(A_{1}-w I\right)^{-1}(A-w I) u .
\end{aligned}
$$

Daher erhalten wir $A_{1}=A$ und somit ist $\left.\lambda \mapsto \hat{R}(\lambda)\right|_{I_{\varkappa}}$ eine verallgemeinerte Resolvente $R$ des Operators $A$.

Wir müssen noch Relation (5.3) zeigen. Dazu seien $x \in \Pi_{x}^{-}, f \in \Pi_{x}$ beliebig. Da $\Pi_{\varkappa}$ dicht in $\Pi_{\varkappa}^{-}$liegt, gibt es eine Folge $\left(f_{n}\right)$ in $\Pi_{\varkappa}$ derart, daß $f_{n} \rightarrow x$ gilt. Demzufolge erhalten wir

$$
[\hat{R}(\lambda) x \mid f]=\lim _{n \rightarrow \infty}\left[R(\lambda) f_{n} \mid f\right]=\lim _{n \rightarrow \infty}\left[f_{n} \mid R(\bar{\lambda}) f\right]=[x \mid R(\bar{\lambda}) f] .
$$

Somit ist $\hat{R}$ eine erweiterte verallgemeinerte Resolvente von $A$.

Universität Jyväskylä

Mathematisches Institut

SF-40100 Jyväskylä 10

Finnland 


\section{Literatur}

[1] Aronszajn, N.: Theory of reproducing kernels. - Trans. Amer. Math. Soc. 68, 1950 , S. $337-404$.

[2] Bergman, S.: The kernel function and conformal mapping. - Mathematical surveys V, American mathematical society, New York, 1950.

[3] De Branges, L.: Some Hilbert spaces of analytic functions. II. - J. Math. Anal. Appl. 11, 1965, S. 44-72.

[4] Iohvidov, I. S., und M. G. KreǏx: Spectral theory of operators in spaces with an indefinite metric. I. - Amer. Math. Soc. Transl. (2) 13, 1960, S. 105-175.

[5] - - - - Spectral theory of operators in spaces with an indefinite metric. II. - Amer. Math. Soc. Transl. (2) 34, 1963, S. 283-373.

[6] Krě̌n, M. G.: Hermitian-positive kernels on homogeneous spaces. I.-II. Amer. Math. Soc. Transl. (2) 34, 1963, S. 69-164.

[7] KreǏn, M. G., und H. LANGER: Defect subspaces and generalized resolvents of an Hermitian operator in the space $\Pi_{\varkappa}$. Functional Anal. Appl. 5, 1971/1972, S. $136-146$ und $217-228$.

[8] - - - - Über die verallgemeinerten Resolventen und die charakteristische Funktion eines isometrischen Operators im Raume $\Pi_{\%}$. Colloquia mathematica Societatis János Bolyai 5: Hilbert space operators and ope. rator algebras [Tihany 1970] (Edited by B. Sz.-Nagy), (Distributed by) North-Holland Publishing Company, Amsterdam / London, 1972, S. $353-399$.

[9] -»- -»- Über die $Q$-Funktion eines $\pi$-hermiteschen Operators im Raume $\Pi_{\varkappa}$. - Acta Sci. Math. (Szeged) 34, 1973, S. 191-230.

[10] Langer, H., und P. SorJonen: Verallgemeinerte Resolventen hermitescher und isometrischer Operatoren im Pontrjaginraum. - Ann. Acad. Sci. Fennicæ A. I. 561, 1974.

[11] Meschkowski, H.: Hilbertsche Räume mit Kernfunktion. - Die Grundlehren der mathematischen Wissenschaften 113, Springer-Verlag, Berlin/ Göttingen/Heidelberg, 1962.

[12] Neumark, M. A.: Normierte Algebren. - Hochschulbücher für Mathematik 45, VEB Deutscher Verlag der Wissenschaften, Berlin, 1959.

[13] Shonkwiler, R.: On generalized resolvents and an integral representation of Nevanlinna. - J. Math. Anal. Appl. 40, 1972, S. 723-734.

[14] SorJonen, P.: Über gewisse Tripel von Pontrjaginräumen. - Math. Nachr. 63, 1974, S. $213-221$.

[15] S̆traus, A. V. [A. В. Штраус]: Обобщенные резольвенты симметріческих операторов. - Изв. Акад. Наук СССР Сер. Мат. 18, 1954, S. 51-86.

[16] Sz.-NAGy, B.: Fortsetzung linearer Transformationen des Hilbertschen Raumes mit Austritt aus dem Raum. - Anhang zu: F. Riesz und B. Sz.-Nagy: Vorlesungen über Funktionalanalysis. [Zweite, berichtigte Auflage.] 
Hochschulbücher für Mathematik 27, VEB Deutscher Verlag der Wissenschaften, Berlin, 1968, S. 427-461.

[17] Sz.-NAGY, B., und C. Foras: Harmonic analysis of operators on Hilbert spaces. Akadémiai Kiadó, Budapest / North-Holland Publishing Company, Amsterdam/London, 1970.

[18] Sz.-NAgY, B., und A. KoránYI: Operatortheoretische Behandlung und Verallge. meinerung eines Problemkreises in der komplexen Funktionentheorie. Acta Math. 100, 1958, S. 171-202. 